\title{
Controlled Loewner-Kufarev Equation Embedded into the Universal Grassmannian
}

\author{
Takafumi $A M A B A^{\dagger}$ and Roland FRIEDRICH ${ }^{\ddagger}$ \\ † Fukuoka University, 8-19-1 Nanakuma, Jônan-ku, Fukuoka, 814-0180, Japan \\ E-mail: fmamaba@fukuoka-u.ac.jp \\ $\ddagger$ ETH Zürich, D-GESS, CH-8092 Zurich, Switzerland \\ E-mail: roland.friedrich@gess.ethz.ch
}

Received June 30, 2020, in final form October 22, 2020; Published online October 29, 2020

https://doi.org/10.3842/SIGMA.2020.108

\begin{abstract}
We introduce the class of controlled Loewner-Kufarev equations and consider aspects of their algebraic nature. We lift the solution of such a controlled equation to the (Sato)-Segal-Wilson Grassmannian, and discuss its relation with the tau-function. We briefly highlight relations of the Grunsky matrix with integrable systems and conformal field theory. Our main result is the explicit formula which expresses the solution of the controlled equation in terms of the signature of the driving function through the action of words in generators of the Witt algebra.
\end{abstract}

Key words: Loewner-Kufarev equation; Grassmannian; conformal field theory; Witt algebra; free probability theory; Faber polynomial; Grunsky coefficient; signature

2020 Mathematics Subject Classification: 35Q99; 30F10; 35C10; 58J65

\section{Introduction}

C. Loewner [21] and P.P. Kufarev [20] initiated a theory which was then further extended by C. Pommerenke [29], and which shows that given any continuously increasing family of simply connected domains containing the origin in the complex plane, the inverses of the Riemann mappings associated to the domains are described by a partial differential equation, the socalled Loewner-(Kufarev) equation

$$
\frac{\partial}{\partial t} f_{t}(z)=z f_{t}^{\prime}(z) p(t, z)
$$

where the $f_{t}$ are the inverses of the Riemann map and $p(z, t)$ is a function with positive real part (see Section 2.2 for details). More recently, I. Markina and A. Vasil'ev [25, 27] considered the so-called alternate Loewner-Kufarev equation, which describes not necessarily increasing chains of domains.

We introduce a further generalisation, namely, the class of controlled Loewner-Kufarev equations

$$
\mathrm{d} f_{t}(z)=z f_{t}^{\prime}(z)\left\{\mathrm{d} x_{0}(t)+\mathrm{d} \xi(\mathbf{x}, z)_{t}\right\}, \quad f_{0}(z) \equiv z \in \mathbb{D},
$$

where $\mathbb{D}$ is the unit disc in the complex plane centred at zero, $x_{0}, x_{1}, x_{2}, \ldots$ are given functions which will be called the driving functions, $\mathbf{x}=\left(x_{1}, x_{2}, \ldots\right)$ and $\xi(\mathbf{x}, z)_{t}:=\sum_{n=1}^{\infty} x_{n}(t) z^{n}$. The controlled Loewner-Kufarev equation can be transformed, after a calculation, into

$$
\mathrm{d} f_{t}(z)=-\sum_{n=0}^{\infty}\left(L_{n} f\right)(z) \mathrm{d} x_{n}
$$


where the $L_{n}:=-z^{n+1} \partial /(\partial z), n \in \mathbb{Z}$, are the generators of the Witt algebra, i.e., the central charge zero Virasoro algebra, satisfying the commutation relations

$$
\left[L_{m}, L_{n}\right]=(m-n) L_{m+n}
$$

Therefore, we are going to consider an extension of [10], where the second author established and studied the role of Lie vector fields, boundary variations and the Witt algebra in connection with the Loewner-Kufarev equation.

Let us recall first some of the classical work of A.A. Kirillov and D.V. Yuriev [15] / G.B. Segal and G. Wilson [32] / N. Kawamoto, Y. Namikawa, A. Tsuchiya and Y. Yamada [14] which will be also fundamental in the present context, in particular in understanding the appearance of the Virasoro algebra with nontrivial central charge.

A.A. Kirillov and D.V. Yuriev [15], constructed a highest weight representation of the Virasoro algebra, where the representation space is given by the space of all holomorphic sections of an analytic line bundle over the orientation-preserving diffeomorphism group Diff $+S^{1}$ of the unit circle $S^{1}$ (modulo rotations). They also gave an embedding of $\left(\operatorname{Diff}_{+} S^{1}\right) / S^{1}$ into the infinite dimensional Grassmannian. In fact, this embedding is an example of a construction of solutions to the KdV hierarchy found by I. Krichever [19], which we address in Section 3.2. If we embed a univalent function on the unit disc $\mathbb{D}$ into the infinite dimensional Grassmannian, by the methods of Kirillov-Yuriev [15], Krichever [19], or Segal-Wilson [32], then one needs to track the Faber polynomials and Grunsky coefficients associated to the univalent function. In general, it is not easy to calculate them from the definition. One of our main results is, however, the following.

Theorem 1.1 (see Propositions 2.12 and 2.14). The Faber polynomials and Grunsky coefficients associated to solutions of the controlled Loewner-Kufarev equation satisfy linear differential equations, and the Grunsky coefficients can be explicitly calculated.

In [10], the second author proposed to lift the embedded Loewner-Kufarev equation to the determinant line bundle over the Sato-Segal-Wilson Grassmannian $\operatorname{Gr}(H)$, as a natural extension of the "Virasoro uniformisation" approach by M. Kontsevich [16] / R. Friedrich and J. Kalkkinen [11] to construct generalised stochastic / Schramm-Loewner evolutions [31] on arbitrary Riemann surfaces, which would also yield a connection with conformal field theory in the spirit of $[14,32]$. Let us also mention the work of B. Doyon [6], who uses conformal loop ensembles (CLE), and which is related to the content of the present article.

In [27], I. Markina and A. Vasil'ev established basic parts of this program, by considering embedded solutions to the Loewner-Kufarev equation into the Segal-Wilson Grassmannian and related the dynamics therein with the representation of the Virasoro algebra, as discussed by Kirillov-Yuriev [15]. Further, they considered the tau-function associated to the embedded solution as a lift to the determinant line bundle. As observed and briefly discussed in $[11,16]$, the generator of the stochastic Loewner equation is hypo-elliptic.

I. Markina, I. Prokhorov and A. Vasil'ev [24] observed and discussed the sub-Riemannian nature of the coefficients of univalent functions. As the second author pointed out [10], this connects with the general theory of hypo-elliptic flows, as explained in the book by F. Baudoin [4], and led him to propose a connection of the (stochastic) Loewner-Kufarev equation with rough paths. Now, in the theory of rough paths (see, e.g., the introduction in [22]), one of the central objects of consideration is the following controlled differential equation:

$$
\mathrm{d} Y_{t}=\varphi\left(Y_{t}\right) \mathrm{d} X_{t}
$$

where $X_{t}$ is a continuous path in a normed space $V$, called the input of (1.2). On the other hand, the path $Y_{t}$ is called the output of (1.2). When we deal with this equation, an important 
object is the signature of the input $X_{t}$, with values in the (extended) tensor algebra associated with $V$ and which is written in the following form:

$$
S(X)_{s, t}:=\left(1, X_{s, t}^{1}, X_{s, t}^{2}, \ldots, X_{s, t}^{n}, \ldots\right), \quad s \leqslant t .
$$

If $X_{t}$ has finite variation with respect to $t$, then each $X_{s, t}^{n}$ is the $n$th iterated integral of $X_{t}$ over the interval $[s, t]$. With this object, a combination of the Magnus expansion and the ChenStrichartz expansion theorem (see, e.g., [4, Section 1.3]) tells us that the output $Y_{t}$ is given as the result of the action of $S(X)_{0, t}$ applied to $Y_{0}$. Heuristically, we may say that a 'group element' $S(X)$ in some big 'group' acts on some element in the (extended) tensor algebra $T((V))$ which gives the output $Y_{t}$, or it might be better to say that the vector field $\varphi$ defines how the 'group element' acts on the algebra. In this spirit, we would like to describe such a picture in the context of controlled Loewner-Kufarev equations.

For this, we extract the algebraic structure of the controlled Loewner-Kufarev equation. If we regard the driving functions $x_{0}, x_{1}, x_{2}, \ldots$ just as letters in an alphabet then it turns out that explicit expressions for the associated Grunsky coefficients are given by the algebra of formal power series, where the space of coefficients is given by words over this alphabet. It is worth mentioning that the action of the words over this alphabet will be actually given by the negative part of the Witt generators. Thus the action of the signature encodes many actions of such negative generators. This can be used to derive a formula for $f_{t}(z)$ as the signature 'applied' to the initial data $f_{0}(z) \equiv z$ (see Theorem 3.8).

Now, given a diffeomorphism of the unit circle $S^{1}$, the solution to the associated conformal welding problem is a solution to the dispersionless Toda lattice hierarchy [34, 36]. Also in this case, the corresponding tau-function is described by the (full) Grunsky coefficients and this generates the solution via an explicit formula. This gives us the possibility to explicitly describe the solution to the conformal welding problem associated to Malliavin's canonic diffusion [23] by means of a controlled Loewner-Kufarev equation; a topic to which we intend to return elsewhere. Since the canonic diffusion is a natural object 'on' the diffeomorphism group of $S^{1}$, as well as the Brownian motion on a Euclidean space, it would describe a natural universal class in the infinite-dimensional situation.

However, the story so far lets us ask how the signature associated with the driving functions describes the corresponding tau-function rather than $f_{t}$ itself.

Theorem 1.2 (see Theorem 3.9). Along the solution of the controlled Loewner-Kufarev equation, the associated tau-function can be written as the determinant of a quadratic form of the signature.

Let us now summarise the structure of the paper. In Section 2, we formulate solutions $f_{t}(z)$ to controlled Loewner-Kufarev equations. We add also a brief review of the classical LoewnerKufarev equation, and then explain how the classical one is recovered from the controlled Loewner-Kufarev equation. We track the variation of the Taylor-coefficients of $f_{t}$ and also the Faber polynomials and Grunsky coefficients. In Section 3, we first recall briefly basics of the Segal-Wilson Grassmannian and Krichever's construction. After that, we describe how a univalent function on $\mathbb{D}$ is embedded into the Grassmannian. We extract the algebraic structure of the controlled Loewner-Kufarev equation in order to obtain Theorem 3.9. In Appendix A, we give the proofs of Theorems 2.10 and 3.9, respectively, and of Proposition 2.14.

\section{The controlled Loewner-Kufarev equation}

General assumption: $\mathbb{N}$ denotes the set of all positive integers, i.e., $\{1,2,3, \ldots\}$, (without zero). 


\subsection{Definition of solutions to controlled Loewner-Kufarev equations}

Given functions $x_{1}, x_{2}, \ldots:[0, T] \rightarrow \mathbb{C}$, we will write $\mathbf{x}:=\left(x_{1}, x_{2}, \ldots\right)$ and

$$
\xi(\mathbf{x}, z)_{t}:=\sum_{n=1}^{\infty} x_{n}(t) z^{n}, \quad \text { for } z \in \mathbb{C}
$$

if it converges. If $A:[0, T] \rightarrow \mathbb{C}$ is of bounded variation, we write $\mathrm{d} A$ or $A(\mathrm{~d} t)$ (when emphasising the coordinate $t$ on $[0, T]$ ) for the associated complex-valued Lebesgue-Stieltjes measure on $[0, T]$, and the total variation measure will be denoted by $|\mathrm{d} A|$.

Definition 2.1. Let $T>0$. Suppose that $x_{0}:[0, T] \rightarrow \mathbb{R}$, as well as $x_{1}, x_{2}, \ldots:[0, T] \rightarrow \mathbb{C}$, are continuous and of bounded variation, and $x_{0}(0)=0$. Let $f_{t}: \mathbb{D} \rightarrow \mathbb{C}$ be conformal mappings for $0 \leqslant t \leqslant T$. We say $\left\{f_{t}\right\}_{0 \leqslant t \leqslant T}$ is a solution to

$$
\mathrm{d} f_{t}(z)=z f_{t}^{\prime}(z)\left\{\mathrm{d} x_{0}(t)+\mathrm{d} \xi(\mathbf{x}, z)_{t}\right\}, \quad f_{0}(z) \equiv z \in \mathbb{D}
$$

if

(1) $f_{0}(z) \equiv z$ for $z \in \mathbb{D}$,

(2) $\sum_{n=1}^{\infty} n \int_{[0, T]}\left|\mathrm{d} x_{n}\right|(t) r^{n}$ converges for all $r \in(0,1)$,

(3) for each compact set $K \subset \mathbb{D}$, the mapping $\left.[0, T] \ni t \mapsto f_{t}^{\prime}\right|_{K} \in C(K)$ is continuous with respect to the uniform norm on $K$,

(4) it holds that

$$
f_{t}(z)-z=\int_{0}^{t} z f_{s}^{\prime}(z)\left\{\mathrm{d} x_{0}(s)+\mathrm{d} \xi(\mathbf{x}, z)_{s}\right\}, \quad(t, z) \in[0, T] \times \mathbb{D} .
$$

In the sequel, we refer to equation (2.1) as a controlled Loewner-Kufarev equation (with driving paths $x_{0}$ and $\left.\mathbf{x}:=\left(x_{1}, x_{2}, \ldots\right)\right)$.

In joint work with T. Murayama [3], we proved that a solution to the controlled Loewner-Kufarev equation is unique if it exists [3, Theorem 3.1]. In the $\omega$-controlled case, for $\omega(0, T)<1 / 2$, a solution exists (and hence uniquely exists), cf. [3, Theorem 3.2]. More specifically, we have

Proposition 2.2 ([3, Lemma 2.1]). Under the assumptions (1)-(3) above,

(i) the series $\xi(\mathbf{x}, z)_{t}$ in $z$ has convergence radius one for each $t \in[0, T]$,

(ii) the family $\{\xi(\mathbf{x}, z)\}_{0 \leqslant t \leqslant T}$ of holomorphic functions on $\mathbb{D}$ is continuous in the topology of locally uniform convergence,

(iii) the function $t \mapsto \xi(\mathbf{x}, z)_{t}$ is of bounded variation and satisfies

$$
\mathrm{d} \xi(\mathbf{x}, z)_{t}=\sum_{k=1}^{\infty} z^{k} \mathrm{~d} x_{k}(t)
$$

for each $z \in \mathbb{D}$.

Furthermore, in [3, equations (3.1) and (3.2)] we proved that $f_{t}^{\prime}(0)=\mathrm{e}^{x_{0}(t)}>0$.

Definition 2.3. We say $\left\{f_{t}\right\}_{0 \leqslant t \leqslant T}$ is a univalent solution to the controlled Loewner-Kufarev equation if it is a solution to (2.1) and $f_{t}$ is a univalent function on $\mathbb{D}$ for each $0 \leqslant t \leqslant T$. 


\subsection{Loewner-Kufarev equation as a controlled Loewner-Kufarev equation}

Definition 2.4. Suppose that $\Omega(t) \subset \mathbb{C}$ is given for each $0 \leqslant t \leqslant T$. $\{\Omega(t)\}_{0 \leqslant t \leqslant T}$ is called a Loewner subordination chain if

(1) $0 \in \Omega(s) \subsetneq \Omega(t)$ for each $0 \leqslant s<t \leqslant T$,

(2) $\Omega(t)$ is a simply connected domain (i.e., open, connected and simply connected) for each $t \in[0, T]$,

(3) (Continuity in the sense of Carathéodory, under the conditions (1) and (2)): For each $t \in[0, T]$ and any sequence $0 \leqslant t_{n} \uparrow t, \cup_{n=1}^{\infty} \Omega\left(t_{n}\right)=\Omega(t)$.

For the following Definition 2.5, cf. specifically [29, Chapter 6, Section 6.1, pp. 156-157; Chapter 2, Section 2.1, p. 35 and Lemma 2.1].

Definition 2.5 ([29]). Let $f_{t}: \mathbb{D} \rightarrow \mathbb{C}$ be given for $0 \leqslant t \leqslant T$. Then $\left\{f_{t}\right\}_{0 \leqslant t \leqslant T}$ is called a Loewner chain if

(1) $f_{t}$ is analytic and univalent on $\mathbb{D}$, for each $0 \leqslant t \leqslant T$,

(2) $f_{t}(z)=\mathrm{e}^{t} z+a_{2}(t) z^{2}+\cdots$, for $z \in \mathbb{D}$,

(3) $f_{s}(\mathbb{D}) \subset f_{t}(\mathbb{D})$, for each $0 \leqslant s<t \leqslant T$.

The above chains $\{\Omega(t)\}$ and $\left\{f_{t}\right\}$ are known to be in one-to-one correspondence via the relation $\Omega(\tau)=f_{t}(\mathbb{D})$, where $t=\log f_{\tau}^{\prime}(0)$ is a time-reparametrisation to satisfy Definition 2.5 $(2)$ (see [29, Chapter 6, Section 6.1]).

Theorem 2.6 ([29, Theorem 6.2]). Let $f_{t}: \mathbb{D} \rightarrow \mathbb{C}$ be given for $0 \leqslant t \leqslant T$. Then $\left\{f_{t}\right\}_{0 \leqslant t \leqslant T}$ is a Loewner chain if and only if there exist constants $r_{0}, K_{0}>0$, and a function $p(t, z)$, analytic in $z \in \mathbb{D}$, and measurable in $t \in[0, T]$ such that

(i) for each $0 \leqslant t \leqslant T$, the function $f_{t}(z)=\mathrm{e}^{t} z+\cdots$ is analytic in $|z|<r_{0}$, the mapping $[0, T] \ni t \mapsto f_{t}(z)$ is absolutely continuous for each $|z|<r_{0}$, and

$$
\left|f_{t}(z)\right| \leqslant K_{0} \mathrm{e}^{t}, \quad \text { for all }|z|<r_{0} \text { and } t \in[0, T] .
$$

(ii) $\operatorname{Re}\{p(t, z)\}>0$, for all $(t, z) \in[0, T] \times \mathbb{D}$, and

$$
\frac{\partial}{\partial t} f_{t}(z)=z f_{t}^{\prime}(z) p(t, z)
$$

for all $|z|<r_{0}$ and for almost all $t \in[0, T]$.

According to the terminology in [5] we call the equation (2.2) the Loewner-Kufarev equation (if we regard $p(t, z)$ as given and $f_{t}(z)$ as unknown).

Because of equation (2.2), it holds that $p(t, 0)=\lim _{z \rightarrow 0}\left(\frac{\partial}{\partial t} f_{t}(z)\right) /\left(z f_{t}^{\prime}(z)\right)=1$, and hence the 'Herglotz representation theorem' applies, which permits us to conclude that, for every $t \in[0, T]$, there exists a probability measure $\nu_{t}$ on $S^{1}=\partial \mathbb{D}$ (which is naturally identified with $[0,2 \pi]$ as measurable spaces, and then the induced probability measure is still denoted by $\nu_{t}$ ) such that

$$
p(t, z)=\int_{0}^{2 \pi} \frac{\mathrm{e}^{i \theta}+z}{\mathrm{e}^{i \theta}-z} \nu_{t}(\mathrm{~d} \theta) \quad \text { for } z \in \mathbb{D} .
$$

Substituting this into (2.2), the Loewner-Kufarev equation becomes

$$
\frac{\partial f_{t}}{\partial t}(z)=z f_{t}^{\prime}(z) \int_{0}^{2 \pi} \frac{\mathrm{e}^{i \theta}+z}{\mathrm{e}^{i \theta}-z} \nu_{t}(\mathrm{~d} \theta) .
$$


Assuming that $\nu_{t}(\mathrm{~d} \theta)=: \nu_{t}(\theta) \mathrm{d} \theta$, we write the Fourier series of $\nu_{t}(\theta)$ as

$$
\nu_{t}(\theta)=\frac{1}{2 \pi}\left\{a_{0}(t)+\sum_{k=1}^{\infty}\left(a_{k}(t) \cos (k \theta)+b_{k}(t) \sin (k \theta)\right)\right\} .
$$

We temporarily introduce the notation $x_{0}(t):=\int_{0}^{t} a_{0}(s) \mathrm{d} s$ and

$$
u_{k}(t):=\int_{0}^{t} a_{k}(s) \mathrm{d} s, \quad v_{k}(t):=-\int_{0}^{t} b_{k}(s) \mathrm{d} s,
$$

for $k=1,2, \ldots$. Because of the relations

$$
\frac{1}{2 \pi} \int_{0}^{2 \pi} \frac{\mathrm{e}^{i \theta}+z}{\mathrm{e}^{i \theta}-z} \cos (k \theta) \mathrm{d} \theta=z^{k}, \quad \frac{1}{2 \pi} \int_{0}^{2 \pi} \frac{\mathrm{e}^{i \theta}+z}{\mathrm{e}^{i \theta}-z} \sin (k \theta) \mathrm{d} \theta=-i z^{k},
$$

for $k=1,2, \ldots$ and $|z|<1$, equation (2.3) assumes the following form:

$$
\frac{\partial f_{t}}{\partial t}(z)=z f_{t}^{\prime}(z)\left\{\dot{x}_{0}(t)+\sum_{k=1}^{\infty}\left(\dot{u}_{k}(t)+i \dot{v}_{k}(t)\right) z^{k}\right\} .
$$

This can be rewritten as the following controlled differential equation

$$
\mathrm{d} f_{t}(z)=z f_{t}^{\prime}(z)\left\{\mathrm{d} x_{0}(t)+\mathrm{d} \xi(\mathbf{x}, z)_{t}\right\},
$$

where $x_{k}(t)=u_{k}(t)+i v_{k}(t)$ for $k \geqslant 1$, and $\xi(\mathbf{x}, z)_{t}=\sum_{k=1}^{\infty} x_{k}(t) z^{k}$.

If we omit the condition $\operatorname{Re}\{p(t, z)\}>0$, that is, we allow the real part of $p(t, z)$ to have an arbitrary sign, then equation (2.2) is called the alternate Loewner-Kufarev equation, as considered by I. Markina and A. Vasil'ev [25]. Intuitively, this describes evolutions of conformal mappings whose images of $\mathbb{D}$ are not necessary increasing, i.e., not strict subordinations. It appears that the general theory with respect to the existence and uniqueness of solutions is not yet fully developed. However, our controlled Loewner-Kufarev equation (2.1) deals with this alternate case because we have not assumed that $p(t, z):=\frac{\mathrm{d}}{\mathrm{d} t}\left(x_{0}(t)+\xi(\mathbf{x}, z)_{t}\right)$ has a positive real part.

Remark 2.7. Readers focusing on radial Loewner equations might feel puzzled by the heuristic assumption that the Radon-Nikodym density $\frac{\nu_{t}(\mathrm{~d} \theta)}{\mathrm{d} \theta}=\nu_{t}(\theta)$ exists, because the radial Loewner equation describes the case $\nu_{t}(\mathrm{~d} \theta)=\delta_{\mathrm{e}^{i w(t)}}(\mathrm{d} \theta)$ where $w(t)$ is a continuous path in $\mathbb{R}$, so that there does not exist a Radon-Nikodym density. However, several explicit examples of LoewnerKufarev equations within this setting, are presented with simulations in Sola [33].

\subsection{Taylor coefficients along the controlled Loewner-Kufarev equation}

Suppose that $x_{0}:[0,+\infty) \rightarrow \mathbb{R}, x_{1}, x_{2}, \ldots:[0,+\infty) \rightarrow \mathbb{C}$ are continuous and of bounded variation. Let $\left\{f_{t}\right\}_{0 \leqslant t \leqslant T}$ be a solution to the controlled Loewner-Kufarev equation (2.1). We parametrise $f_{t}$ as

$$
f_{t}(z)=C(t)\left(z+c_{1}(t) z^{2}+c_{2}(t) z^{3}+c_{3}(t) z^{4}+\cdots\right),
$$

with the additional convention that $c_{0}(t) \equiv 1$.

The dynamics of the coefficients $\left(c_{1}, c_{2}, \ldots\right)$ has been previously studied by Vasil'ev and his co-authors [12, 24, 25, 26]. The (stochastic/Schramm)-Loewner (equation/evolution) (SLE) case is discussed by Friedrich [10]. A complementary, conformal field theoretic perspective of the Bieberbach-de Branges theorem is given by Duplantier et al. [7]. Within our framework, we get the following similarly: 
Proposition 2.8. Let $\left\{f_{t}\right\}_{0 \leqslant t \leqslant T}$ be a solution to the controlled Loewner-Kufarev equation (2.1) with the parametrisation (2.4). Then we have

$$
\mathrm{d} C(t)=C(t) \mathrm{d} x_{0}(t),
$$

and

$$
\left\{\begin{aligned}
& \mathrm{d} c_{1}(t)=\mathrm{d} x_{1}(t)+ c_{1}(t) \mathrm{d} x_{0}(t), \\
& \mathrm{d} c_{2}(t)=\mathrm{d} x_{2}(t)+ 2 c_{1}(t) \mathrm{d} x_{1}(t)+2 c_{2}(t) \mathrm{d} x_{0}(t), \\
& \vdots \\
& \mathrm{d} c_{n}(t)=\mathrm{d} x_{n}(t)+\sum_{k=1}^{n-1}(k+1) c_{k}(t) \mathrm{d} x_{n-k}(t)+n c_{n}(t) \mathrm{d} x_{0}(t), \quad \text { for } n \geqslant 2,
\end{aligned}\right.
$$

with the initial conditions $C(0)=1$ and $c_{1}(0)=c_{2}(0)=\cdots=0$. In particular, $C=\{C(t)\}_{0 \leqslant t \leqslant T}$ takes its values in $\mathbb{R}$.

As $f_{t}^{\prime}(0)=C(t)=\mathrm{e}^{x_{0}(t)-x_{0}(0)} \neq 0$, we get

Corollary 2.9. Let $\left\{f_{t}\right\}_{0 \leqslant t \leqslant T}$ be a solution to the controlled Loewner-Kufarev equation (2.1). Then $f_{t}$ is univalent in a neighbourhood of 0 , for each $0 \leqslant t \leqslant T$.

Theorem 2.10. Let $\left\{f_{t}\right\}_{0 \leqslant t \leqslant T}$ be a solution to the controlled Loewner-Kufarev equation (2.1). Then for each $n \in \mathbb{N}$, the coefficient $c_{n}$ in (2.4) is given by

$$
\begin{aligned}
c_{n}(t)= & \sum_{p=1}^{n} \sum_{\substack{i_{1}, \ldots, i_{p} \in \mathbb{N}: \\
i_{1}+\cdots+i_{p}=n}} \widetilde{w}(n)_{i_{1}, \ldots, i_{p}} \mathrm{e}^{n x_{0}(t)} \\
& \times \int_{0 \leqslant s_{1}<s_{2}<\cdots<s_{p} \leqslant t} \mathrm{e}^{-i_{1} x_{0}\left(s_{1}\right)} \mathrm{d} x_{i_{1}}\left(s_{1}\right) \mathrm{e}^{-i_{2} x_{0}\left(s_{2}\right)} \mathrm{d} x_{i_{2}}\left(s_{2}\right) \cdots \mathrm{e}^{-i_{p} x_{0}\left(s_{p}\right)} \mathrm{d} x_{i_{p}}\left(s_{p}\right),
\end{aligned}
$$

where

$$
\widetilde{w}(n)_{i_{1}, \ldots, i_{p}}:=\left\{\left(n-i_{1}\right)+1\right\}\left\{\left(n-\left(i_{1}+i_{2}\right)\right)+1\right\} \cdots\left\{\left(n-\left(i_{1}+i_{2}+\cdots+i_{p-1}\right)\right)+1\right\},
$$

and $n=i_{1}+\cdots+i_{p}$.

The proof can be found in Appendix A.1.

\subsection{Variation of Grunsky coefficients induced by a Loewner-Kufarev equation}

There are several different ways to introduce the Faber polynomials. Here we give a derivation by utilising Teo [35], and, an alternative one, in Section 3.3, which serves our purpose better. For a (formal) power series $f(z)=a_{1} z+a_{2} z^{2}+a_{3} z^{3}+\cdots, a_{1} \neq 0$, the (generalised) Faber polynomials $Q_{n}(w), n \in \mathbb{N}$, associated to $f$, are defined as

$$
\log \frac{w-f(z)}{w}=\log \frac{f(z)}{a_{1} z}-\sum_{n=1}^{\infty} \frac{Q_{n}(w)}{n} z^{n} .
$$

By differentiating equation (2.6), and reordering it, we obtain, via the Residue theorem, the Faber polynomials (cf. also expression (3.1)), as

$$
Q_{n}(w)=\operatorname{Res}_{z=0}\left[\frac{w z^{-n}}{w-f(z)} \frac{f^{\prime}(z)}{f(z)}\right] \mathrm{d} z \underset{\zeta=f(z)}{=} \operatorname{Res}_{\zeta=0}\left[\frac{\left(f^{-1}(\zeta)\right)^{-n}}{1-\zeta w^{-1}} \frac{1}{\zeta}\right] \mathrm{d} \zeta .
$$


The coefficients $\left(b_{-m,-n}\right)_{m, n=1}^{\infty}$ in the series expansion

$$
\log \frac{f(z)-f(\zeta)}{z-\zeta}=-\sum_{m=0}^{\infty} \sum_{n=0}^{\infty} b_{-m,-n} z^{m} \zeta^{n},
$$

at $(z, \zeta)=(0,0)$, are called the (generalised) Grunsky coefficients of $f$. Equivalently, these are defined via the Laurent series at $z=0$,

$$
Q_{n}(f(z))=z^{-n}+n \sum_{m=1}^{\infty} b_{-n,-m} z^{m} .
$$

Proposition 2.11. Let $\left\{f_{t}\right\}_{0 \leqslant t \leqslant T}$ be a solution to the controlled Loewner-Kufarev equation (2.1). Then there exists an open neighbourhood $U$ of the origin, such that

(i) $\bar{U} \subset \mathbb{D}$,

(ii) $\left.f_{t}\right|_{U}$ is univalent for each $t \in[0, T]$,

(iii) $V:=\bigcap_{0 \leqslant t \leqslant T} f_{t}(U)$ is an open neighbourhood of the origin,

(iv) for each $\zeta \in V,[0, T] \ni t \mapsto f_{t}^{-1}(\zeta)$ is continuous and of bounded variation,

$(v)$ for each $\zeta \in V$, with $f^{-1}(t, \zeta):=f_{t}^{-1}(\zeta)$ and $\mathrm{d} f_{t}^{-1}(\zeta):=f^{-1}(\mathrm{~d} t, \zeta)$, we have

$$
\mathrm{d} f_{t}^{-1}(\zeta)=-f_{t}^{-1}(\zeta)\left\{\mathrm{d} x_{0}(t)+\sum_{k=1}^{\infty}\left(f_{t}^{-1}(\zeta)\right)^{k} \mathrm{~d} x_{k}(t)\right\}
$$

as Lebesgue-Stieltjes measures on $[0, T]$.

Let $\left\{f_{t}\right\}_{0 \leqslant t \leqslant T}$ be a solution to the controlled Loewner-Kufarev equation (2.1). Because of Corollary 2.9, associated to each $f_{t}(z)$ are the corresponding Faber polynomials and Grunsky coefficients, which will be denoted by $Q_{n}(t, w)$, and $b_{-n,-m}(t)$, respectively.

\section{Proposition 2.12.}

(i) (Variation of Faber polynomials): We have for each $n \in \mathbb{N}$,

$$
\mathrm{d} Q_{n}(t, w)=n \mathrm{~d} x_{n}(t)+n \sum_{k=1}^{n} Q_{k}(t, w) \mathrm{d} x_{n-k}(t) .
$$

(ii) (Variation of Grunsky coefficients): For each $n, m \in \mathbb{N}$,

$$
\begin{aligned}
\mathrm{d} b_{-n,-m}(t)= & -\mathrm{d} x_{n+m}(t)+\sum_{\substack{k, l \in \mathbb{Z}_{\geqslant 0} ; \\
k+l=m-1}}(k+1) b_{-n,-(k+1)}(t) \mathrm{d} x_{l}(t) \\
& +\sum_{\substack{k, l \in \mathbb{Z}_{\geqslant 0} ; \\
k+l=n-1}}(k+1) b_{-m,-(k+1)} \mathrm{d} x_{l}(t),
\end{aligned}
$$

with the initial condition $b_{-n,-m}(0)=0$, for all $n, m \in \mathbb{N}$.

Proof. (i) Let $n \in \mathbb{N}$. Let $U$ and $V$ be as in Proposition 2.11. Then $f_{t}^{-1}(\zeta), \zeta \in V$, satisfies the equation

$$
\mathrm{d} f_{t}^{-1}(\zeta)=-f_{t}^{-1}(\zeta)\left\{\mathrm{d} x_{0}(t)+\sum_{k=1}^{\infty}\left(f_{t}^{-1}(\zeta)\right)^{k} \mathrm{~d} x_{k}(t)\right\} .
$$


Let $X_{0} \subset V$ be an open disc centred at 0 . By using Cauchy's integral formula, we have for $w \in X_{0}$,

$$
\begin{aligned}
\mathrm{d} Q_{n}(t, w) & =\frac{1}{2 \pi i} \int_{\partial X_{0}} \frac{\mathrm{d} \zeta}{\zeta} \frac{\mathrm{d}\left(f_{t}^{-1}(\zeta)\right)^{-n}}{1-\zeta w^{-1}} \\
& =\frac{1}{2 \pi i} \int_{\partial X_{0}}(-n) \frac{\left(f_{t}^{-1}(\zeta)\right)^{-n-1}}{1-\zeta w^{-1}}\left(-f_{t}^{-1}(\zeta)\right) \sum_{k=0}^{\infty}\left(f_{t}^{-1}(\zeta)\right)^{k} \mathrm{~d} x_{k}(t) \frac{\mathrm{d} \zeta}{\zeta} \\
& =\sum_{k=0}^{n} \frac{n}{2 \pi i}\left(\int_{\partial X_{0}} \frac{\left(f_{t}^{-1}(\zeta)\right)^{-n+k}}{1-\zeta w^{-1}} \frac{\mathrm{d} \zeta}{\zeta}\right) \mathrm{d} x_{k}(t) \\
& =\frac{n \mathrm{~d} x_{n}(t)}{2 \pi i} \int_{\partial X_{0}} \frac{1}{1-\zeta w^{-1}} \frac{\mathrm{d} \zeta}{\zeta}+n \sum_{k=0}^{n-1} Q_{n-k}(t, w) \mathrm{d} x_{k}(t) .
\end{aligned}
$$

By noting that the orientation of $\partial X_{0}$ is anti-clockwise, we get

$$
\frac{1}{2 \pi i} \int_{\partial X_{0}} \frac{1}{1-\zeta w^{-1}} \frac{\mathrm{d} \zeta}{\zeta}=1
$$

and hence the result.

(ii) By putting $p(\mathrm{~d} t, z):=\mathrm{d} x_{0}(t)+\mathrm{d} \xi(\mathbf{x}, z)_{t}$, and since $f_{t}(z)$ satisfies the controlled LoewnerKufarev equation, we have

$$
\begin{aligned}
\mathrm{d} Q_{n}\left(t, f_{t}(z)\right) & =Q_{n}\left(\mathrm{~d} t, f_{t}(z)\right)+Q_{n}^{\prime}\left(t, f_{t}(z)\right) \mathrm{d} f_{t}(z) \\
& =Q_{n}\left(\mathrm{~d} t, f_{t}(z)\right)+Q_{n}^{\prime}\left(t, f_{t}(z)\right)\left\{z f_{t}^{\prime}(z) p(\mathrm{~d} t, z)\right\} \\
& =Q_{n}\left(\mathrm{~d} t, f_{t}(z)\right)+z\left[\partial_{z} Q_{n}\left(t, f_{t}(z)\right)\right] p(\mathrm{~d} t, z),
\end{aligned}
$$

so that

$$
\mathrm{d} Q_{n}\left(t, f_{t}(z)\right)=Q_{n}\left(\mathrm{~d} t, f_{t}(z)\right)+z\left[\partial_{z} Q_{n}\left(t, f_{t}(z)\right)\right] p(\mathrm{~d} t, z) .
$$

By recalling that $Q_{n}\left(t, f_{t}(z)\right)=z^{-n}+n \sum_{m=1}^{\infty} b_{-n,-m}(t) z^{m}$, we have, by substitution, the following sequence of identities

$$
(\text { LHS of }(2.9))_{\geqslant 1}=(\text { LHS of }(2.9))=n \sum_{m=1}^{\infty} z^{m} \mathrm{~d} b_{-n,-m}(t) .
$$

Here, $(\cdots)_{\geqslant 1}$ is the operator which forgets those terms in $(\cdots)$, whose degree is less than one. On the other hand, by Proposition 2.12(i), we have

$$
\begin{aligned}
\mathrm{d} Q_{n}\left(t, f_{t}(z)\right) & =n \mathrm{~d} x_{n}(t)+n \sum_{k=1}^{n} Q_{k}\left(t, f_{t}(z)\right) \mathrm{d} x_{n-k}(t) \\
& =n \mathrm{~d} x_{n}(t)+n \sum_{k=1}^{n}\left(z^{-k}+k \sum_{m=1}^{\infty} b_{-k,-m}(t) z^{m}\right) \mathrm{d} x_{n-k}(t) \\
& =n \mathrm{~d} x_{n}(t)+n \sum_{k=1}^{n} z^{-k} \mathrm{~d} x_{n-k}(t)+n \sum_{m=1}^{\infty}\left(\sum_{k=1}^{n} k b_{-k,-m}(t) \mathrm{d} x_{n-k}(t)\right) z^{m},
\end{aligned}
$$

so that

$$
\left(\mathrm{d} Q_{n}\left(t, f_{t}(z)\right)\right)_{\geqslant 1}=n \sum_{m=1}^{\infty}\left(\sum_{k=1}^{n} k b_{-k,-m} \mathrm{~d} x_{n-k}(t)\right) z^{m} .
$$


We further have

$$
\begin{aligned}
z\left[\partial_{z} Q_{n}\left(t, f_{t}(z)\right)\right] p(\mathrm{~d} t, z)= & z\left(-n z^{-n-1}+n \sum_{k=1}^{\infty} k b_{-n,-k} z^{k-1}\right)\left(\mathrm{d} x_{0}(t)+\sum_{l=1}^{\infty} \mathrm{d} x_{l}(t) z^{l}\right) \\
= & n\left(-\mathrm{d} x_{0}(t) z^{-n}-\sum_{m=1-n}^{\infty} \mathrm{d} x_{m+n}(t) z^{m}\right. \\
& \left.+\sum_{m=1}^{\infty} m b_{-n,-m}(t) \mathrm{d} x_{0}(t) z^{m}+\sum_{m=2}^{\infty} \sum_{\substack{k, l \geqslant 1 ; \\
k+l=m}} k b_{-n,-k}(t) \mathrm{d} x_{l}(t) z^{m}\right),
\end{aligned}
$$

from which we conclude

$$
\left(z\left[\partial_{z} Q_{n}\left(t, f_{t}(z)\right)\right] p(\mathrm{~d} t, z)\right)_{\geqslant 1}=n \sum_{m=1}^{\infty}\left(-\mathrm{d} x_{n+m}(t)+\sum_{\substack{k \geqslant 1, l \geqslant 0 ; \\ k+l=m}} k b_{-n,-k}(t) \mathrm{d} x_{l}(t)\right) z^{m} .
$$

Combining (2.11) and (2.12), we obtain

$$
\begin{aligned}
(\text { RHS of }(2.9))_{\geqslant 1}= & n \sum_{m=1}^{\infty}\left(-\mathrm{d} x_{n+m}(t)+\sum_{\substack{k, l \in \mathbb{Z}_{\geqslant 0} ; \\
k+l=m-1}}(k+1) b_{-n,-(k+1)}(t) \mathrm{d} x_{l}(t)\right. \\
& \left.+\sum_{\substack{k, l \in \mathbb{Z}_{\geqslant 0} ; \\
k+l=n-1}}(k+1) b_{-m,-(k+1)} \mathrm{d} x_{l}(t)\right) z^{m},
\end{aligned}
$$

and then by comparing with (2.10), we get the result. Furthermore, the initial condition is derived from $f_{0}(z) \equiv z$.

In order to derive an explicit formula for the Grunsky coefficients $b_{-n,-m}(t)$, cf. equation (2.7), we shall introduce some notation. In [2], we study analytic aspects of these coefficients.

Definition 2.13. Let $p, q \in \mathbb{N}$.

(1) A bijection $\sigma:\{1,2, \ldots, p+q\} \rightarrow\{1,2, \ldots, p+q\}$ is called a $(p, q)$-shuffle if it holds that $\sigma(1)<\sigma(2)<\cdots<\sigma(p)$ and $\sigma(p+1)<\sigma(p+2)<\cdots<\sigma(p+q)$.

(2) Suppose that $x_{1}, x_{2}, \ldots, x_{p+q}:[0, T] \rightarrow \mathbb{C}$ are continuous and of bounded variation. Then for each $0 \leqslant t \leqslant T$, we set

$$
\begin{aligned}
\left(\left(x_{1} \cdots x_{p}\right)\right. & \text { W } \left.\left(x_{p+1} \cdots x_{p+q}\right)\right)(t) \\
& :=\int_{0 \leqslant s_{q} \leqslant \cdots \leqslant s_{1} \leqslant t_{p} \leqslant \cdots \leqslant t_{1} \leqslant t}\left(\mathrm{~d} x_{1}\left(t_{1}\right) \cdots \mathrm{d} x_{p}\left(t_{p}\right)\right) \amalg\left(\mathrm{d} x_{p+1}\left(s_{1}\right) \cdots \mathrm{d} x_{p+q}\left(s_{q}\right)\right) \\
& :=\sum_{\sigma^{-1}:} \int_{(p, q) \text {-shuffle }}^{t} \mathrm{~d} x_{\sigma(1)}\left(t_{1}\right) \int_{0}^{t_{1}} \mathrm{~d} x_{\sigma(2)}\left(t_{2}\right) \cdots \int_{0}^{t_{p-1}} \mathrm{~d} x_{\sigma(p)}\left(t_{p}\right) \\
& \times \int_{0}^{t_{p}} \mathrm{~d} x_{\sigma(p+1)}\left(s_{1}\right) \int_{0}^{s_{1}} \mathrm{~d} x_{\sigma(p+2)}\left(s_{2}\right) \cdots \int_{0}^{s_{q-1}} \mathrm{~d} x_{\sigma(p+q)}\left(s_{q}\right) .
\end{aligned}
$$

The general formula for the Grunsky-coefficients along the controlled Loewner-Kufarev equation (2.1) is stated as next, and which is crucial for the embedding into the Grassmannian, cf. Section 3. The proof is given in Appendix A.2. 
Proposition 2.14. For $n, m \in \mathbb{N}$ and $t \geqslant 0$,

$$
\begin{aligned}
& b_{-m,-n}(t)=-\mathrm{e}^{(n+m) x_{0}(t)} \int_{0}^{t} \mathrm{e}^{-(n+m) x_{0}(s)} \mathrm{d} x_{m+n}(s)
\end{aligned}
$$

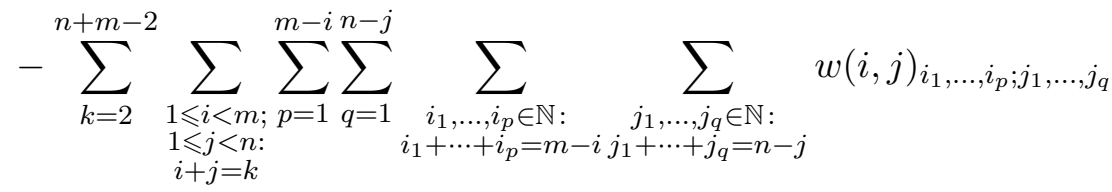

$$
\begin{aligned}
& \times \mathrm{e}^{(m+n) x_{0}(t)} \int_{0 \leqslant u_{q} \leqslant \cdots \leqslant u_{1} \leqslant s_{q} \leqslant \cdots \leqslant s_{1} \leqslant t}\left(\mathrm{e}^{-i_{1} x_{0}\left(s_{1}\right)} \mathrm{d} x_{i_{1}}\left(s_{1}\right) \cdots \mathrm{e}^{-i_{p} x_{0}\left(s_{p}\right)} \mathrm{d} x_{i_{p}}\left(s_{p}\right)\right) \\
& \varpi\left(\mathrm{e}^{-j_{1} x_{0}\left(u_{1}\right)} \mathrm{d} x_{j_{1}}\left(u_{1}\right) \cdots \mathrm{e}^{-j_{q} x_{0}\left(u_{q}\right)} \mathrm{d} x_{j_{q}}\left(u_{q}\right)\right) \int_{0}^{u_{q}} \mathrm{e}^{-k x_{0}(s)} \mathrm{d} x_{k}(s) \\
& -\sum_{k=m+1}^{n+m-1} \sum_{q=1}^{n+m-k} \sum_{\substack{j_{1}, \ldots, j_{q} \in \mathbb{N}: \\
j_{1}+\cdots+j_{q}=n+m-k}} w(k-m)_{\varnothing ; j_{1}, \ldots, j_{q}} \\
& \times \mathrm{e}^{(m+n) x_{0}(t)} \int_{0 \leqslant s_{q} \leqslant \cdots \leqslant s_{1} \leqslant t}\left(\mathrm{e}^{-j_{1} x_{0}\left(s_{1}\right)} \mathrm{d} x_{j_{1}}\left(s_{1}\right) \cdots \mathrm{e}^{-j_{q} x_{0}\left(s_{q}\right)} \mathrm{d} x_{j_{q}}\left(s_{q}\right)\right) \\
& \times \int_{0}^{s_{q}} \mathrm{e}^{-k x_{0}(s)} \mathrm{d} x_{k}(s)-\sum_{k=n+1}^{n+m-1} \sum_{p=1}^{m+n-k} \sum_{\substack{i_{1}, \ldots, i_{p} \in \mathbb{N}: \\
i_{1}+\cdots+i_{p}=m+n-k}} w(k-n)_{i_{1}, \ldots, i_{p} ; \varnothing} \\
& \times \mathrm{e}^{(m+n) x_{0}(t)} \int_{0 \leqslant u_{p} \leqslant \cdots \leqslant u_{1} \leqslant t}\left(\mathrm{e}^{-i_{1} x_{0}\left(u_{1}\right)} \mathrm{d} x_{i_{1}}\left(u_{1}\right) \cdots \mathrm{e}^{-i_{p} x_{0}\left(u_{p}\right)} \mathrm{d} x_{i_{p}}\left(u_{p}\right)\right) \\
& \times \int_{0}^{u_{p}} \mathrm{e}^{-k x_{0}(u)} \mathrm{d} x_{k}(u)
\end{aligned}
$$

where, for $m=i_{1}+\cdots+i_{p}+r$, and $n=j_{1}+\cdots+j_{q}+s$, we have put

$$
\begin{aligned}
& w(r)_{i_{1}, \ldots, i_{p} ; \varnothing}=\left(m-i_{1}\right)\left(m-\left(i_{1}+i_{2}\right)\right) \cdots\left(m-\left(i_{1}+i_{2}+\cdots+i_{p}\right)\right), \\
& w(s)_{\varnothing ; j_{1}, \ldots, j_{q}}=\left(n-j_{1}\right)\left(n-\left(j_{1}+j_{2}\right)\right) \cdots\left(n-\left(j_{1}+j_{2}+\cdots+j_{q}\right)\right),
\end{aligned}
$$

and $w(r, s)_{i_{1}, \ldots, i_{p} ; j_{1}, \ldots, j_{q}}:=w(r)_{i_{1}, \ldots, i_{p} ; \varnothing} w(s)_{\varnothing ; j_{1}, \ldots, j_{q}} \cdot$

\section{The controlled Loewner-Kufarev equation embedded into the Segal-Wilson Grassmannian}

\subsection{Segal-Wilson Grassmannian}

Let $H:=L^{2}\left(S^{1}, \mathbb{C}\right)$ be the Hilbert space which consists of all square-integrable complex functions on the unit circle $S^{1}$. It decomposes orthogonally into $H=H_{+} \oplus H_{-}$, where $H_{+}$and $H_{-}$ are the closure of $\operatorname{span}\left\{z^{k}: k \geqslant 0\right\}$ and $\operatorname{span}\left\{z^{k}: k<0\right\}$, respectively.

Definition 3.1 (G. Segal and G. Wilson [32, Section 2]). The Segal-Wilson Grassmannian $\mathrm{Gr}:=\operatorname{Gr}(H)$ is the set of all closed subspaces $W$ of $H$ satisfying the following:

(1) The orthogonal projection $\mathrm{pr}_{+}: W \rightarrow H_{+}$is Fredholm,

(2) The orthogonal projection $\mathrm{pr}_{-}: W \rightarrow H_{-}$is compact. 
The Fredholm index of the orthogonal projection $\mathrm{pr}_{+}: W \rightarrow H_{+}$is called the virtual dimension of $W$. For $d \in \mathbb{Z}$, we set

$\operatorname{Gr}\left(\frac{\infty}{2}+d, \infty\right):=\{W \in \mathrm{Gr}$ : the virtual dimension of $W$ is $d\}$,

and $\operatorname{Gr}\left(\frac{\infty}{2}, \infty\right):=\operatorname{Gr}\left(\frac{\infty}{2}+0, \infty\right)$.

If we take $W=H_{+}$, then the corresponding projections are given by $\mathrm{pr}_{+}=\mathrm{id}_{H_{+}}$and $\mathrm{pr}_{-}=0$, which are Fredholm and compact operators, respectively. Therefore we have $H_{+} \in \operatorname{Gr}\left(\frac{\infty}{2}, \infty\right)$.

Definition 3.2 ([32, Section 5]). Let $\Gamma_{+}$denote the set of all continuous functions $g: S^{1} \rightarrow \mathbb{C}^{*}$, such that $g(z)=\mathrm{e}^{\sum_{k=1}^{\infty} t_{k} z^{k}}, z \in S^{1}$ for some $\mathbf{t}=\left(t_{1}, t_{2}, t_{3}, \ldots\right)$.

The set $\Gamma_{+}$acts on $H$ by pointwise multiplication. In particular, $\Gamma_{+}$forms a group. This action induces the action of $\Gamma_{+}$on $\mathrm{Gr}: \Gamma_{+} \times \mathrm{Gr} \ni(g, W) \mapsto g W \in \mathrm{Gr}$ (see [32, Lemma 2.2 and Proposition 2.3]), where $g W=\{g f: f \in W\}$. For any $g=\mathrm{e}^{\sum_{k=1}^{\infty} t_{k} z^{k}} \in \Gamma_{+}$, the action of $g$ on $H$ is of the form

$$
g=\left(\begin{array}{ll}
a & b \\
0 & d
\end{array}\right) \quad \text { along } H=H_{+} \oplus H_{-},
$$

where $a: H_{+} \rightarrow H_{+}$is invertible and $b: H_{-} \rightarrow H_{+}$is of trace class (see [32, Proposition 2.3]). Let $\mathcal{U}$ be the set of all $W \in \operatorname{Gr}\left(\frac{\infty}{2}, \infty\right)$ such that the orthogonal projection $W \rightarrow H_{+}$is an isomorphism. Then, associated to each $W \in \mathcal{U}$ is the tau-function $\tau_{W}(\mathbf{t})$ of $W$, a function of infinitely many "times" $\mathbf{t}=\left(t_{1}, t_{2}, \ldots\right)$. It is known that the following holds:

Proposition 3.3 ([32, Proposition 3.3]). Let $W \in \mathcal{U}$. For $g=\mathrm{e}^{\sum_{n=1}^{\infty} t_{n} z^{n}} \in \Gamma_{+}$, we have

$$
\tau_{W}(\mathbf{t})=\operatorname{det}\left(1+a^{-1} b A\right),
$$

where $\mathbf{t}=\left(t_{1}, t_{2}, t_{3}, \ldots\right)$,

$$
g^{-1}=\left(\begin{array}{ll}
a & b \\
0 & d
\end{array}\right) \quad \text { along } H=H_{+} \oplus H_{-},
$$

and $A: H_{+} \rightarrow H_{-}$is the linear operator such that $\operatorname{graph}(A)=W$.

\subsection{Krichever's construction}

In connection with algebraic geometry and infinite-dimensional integrable systems, a fundamental observation / construction of Krichever [17, 18, 19] states the following. A solution of the $\mathrm{KdV}$ equation is associated with each non-singular algebraic curve, equipped with some additional algebro-geometric data. Segal and Wilson [32] developed and formalised, after a remark by Mumford [28], this construction further.

The specific algebro-geometric datum is given by a quintuple $\left(X, \mathcal{L}, x_{\infty}, z, \varphi\right)$, consisting of the following parts. $X$ is a complete, irreducible and complex algebraic curve with a rankone, torsion-free coherent sheaf $\mathcal{L}$. Additionally, a non-singular point $x_{\infty} \in X$, and a closed neighbourhood $X_{\infty}$, are chosen, such that there exists a local parameter $1 / z: X_{\infty} \rightarrow \overline{\mathbb{D}} \subset \widehat{\mathbb{C}}$, with $x_{\infty} \mapsto 0$, and a trivialisation $\varphi:\left.\mathcal{L}\right|_{X_{\infty}} \rightarrow \overline{\mathbb{D}} \times \mathbb{C}$, of $\left.\mathcal{L}\right|_{X_{\infty}}$. Each section of $\left.\mathcal{L}\right|_{X_{\infty}}$ is identified with a complex function on $\overline{\mathbb{D}}$ under $\varphi$. For $X_{0}:=X \backslash X_{\infty}^{\circ}$, with $X_{\infty}^{\mathrm{o}}$ the interior of $X_{\infty}$, the closed sets $X_{0}$ and $X_{\infty}$ cover $X$, and $X_{0} \cap X_{\infty}$ is identified with $S^{1}$ under $z$.

Given this algebro-geometric datum, one can associate a closed subspace $W \subset H$, consisting of all analytic functions $S^{1} \rightarrow \mathbb{C}$ which, under the above identification, extend to a holomorphic section of $\mathcal{L}$ on an open neighbourhood of $X_{0}$. More explicitly, one can write

$$
W=\left\{\begin{array}{c}
\text { the second component } \\
\text { of }\left.\varphi \circ s \circ(1 / z)^{-1}\right|_{S^{1}}: \begin{array}{l}
\text { is a holomorphic section } \\
\text { on a neighbourhood of } X_{0}
\end{array}
\end{array}{ }^{H},\right.
$$


where $(1 / z)^{-1}: \overline{\mathbb{D}} \rightarrow X_{\infty}$ is the inverse function of $1 / z$. It is known that $W \in$ Gr (see [32, Proposition 6.1]), and if $X$ is a compact Riemann surface (then $\mathcal{L}$ is automatically a complex line bundle, hence a maximal torsion-free sheaf), this correspondence $\left(X, \mathcal{L}, x_{\infty}, z, \varphi\right) \mapsto W \in \mathrm{Gr}$ is one-to-one (see [32, Proposition 6.2]).

\subsection{The appearance of Faber polynomials and Grunsky coefficients}

Let $f: \mathbb{D} \rightarrow \mathbb{C}$ be a univalent function such that $f(0)=0$, and $f(\mathbb{D})$ is bounded by a Jordan curve. We set $\beta: \widehat{\mathbb{C}} \rightarrow \widehat{\mathbb{C}}$ by $\beta(w):=1 / w$. For a subset $A \subset \widehat{\mathbb{C}}$, we shall write $A^{-1}:=\beta(A)$, and let $\widehat{\mathbb{D}}_{\infty}:=\widehat{\mathbb{C}} \backslash \overline{\mathbb{D}}$. We obtain an algebro-geometric datum $\left(X, \mathcal{L}, x_{\infty}, z, \varphi\right)$ by setting $X=\widehat{\mathbb{C}}$, $\mathcal{L}=\widehat{\mathbb{C}} \times \mathbb{C}, x_{\infty}:=\infty, X_{\infty}:=f(\overline{\mathbb{D}})^{-1}, z:=\beta \circ f^{-1} \circ \beta^{-1}: X_{\infty} \rightarrow \widehat{\mathbb{D}}_{\infty}$, and $\varphi=(1 / z) \times \mathrm{id}_{\mathbb{C}}$. Correspondingly, we have $X_{0}=\widehat{\mathbb{C}} \backslash\left(f(\mathbb{D})^{-1}\right)$. Further, by the Caratheodory extension theorem, $z$ extends continuously to $X_{\infty}$, and therefore we can embed $f$, by assigning a Hilbert space $W=W_{f}$ to it, into the Grassmannian. In this case, we have $\widehat{\mathbb{C}} \backslash\left(f(\mathbb{D})^{-1}\right)$, and hence

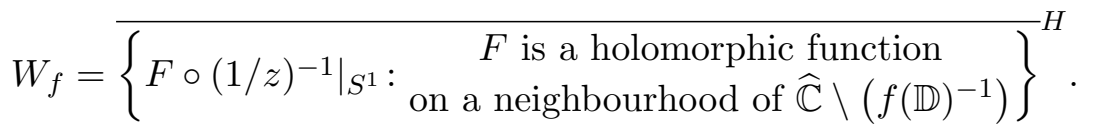

In order to start this paper's main calculation, let us specify this more explicitly. For a closed subset $V$ in $\widehat{\mathbb{C}}$, we denote by $\mathcal{O}(V)$ the space of all holomorphic functions defined on an open neighbourhood of $V$. For a univalent function $g: \widehat{\mathbb{D}}_{\infty} \rightarrow \widehat{\mathbb{C}}$, with $g(\infty)=\infty$, and for each $h \in \mathcal{O}(\overline{\mathbb{D}})$, we call

$$
(\mathcal{F}[h])(z):=\frac{1}{2 \pi i} \int_{\partial\left(\mathbb{C} \backslash g\left(\mathbb{D}_{\infty}\right)\right)} \frac{h\left(g^{-1}(\xi)\right)}{\xi-z} \mathrm{~d} \xi, \quad z \in \mathbb{C} \backslash \overline{g\left(\mathbb{D}_{\infty}\right)}
$$

the Faber transform of $h$ (with respect to $g$ ). If the boundary $\partial\left(\mathbb{C} \backslash g\left(\mathbb{D}_{\infty}\right)\right.$ ) is analytic, it is known that $h \in \mathcal{O}(\overline{\mathbb{D}})$ iff $\mathcal{F} h \in \mathcal{O}\left(\mathbb{C} \backslash g\left(\mathbb{D}_{\infty}\right)\right.$ ) (see $\left[13\right.$, Theorem 1]) and $\mathcal{F}: \mathcal{O}(\overline{\mathbb{D}}) \rightarrow \mathcal{O}\left(\mathbb{C} \backslash g\left(\mathbb{D}_{\infty}\right)\right.$ ) is bijective. In our case, we put

$$
g:=(1 / z)^{-1}=\beta \circ f \circ \beta^{-1}: \widehat{\mathbb{D}}_{\infty} \rightarrow f(\mathbb{D})^{-1},
$$

and then we can describe $\mathcal{O}\left(X_{0}\right)$ by $\mathcal{O}\left(\widehat{\mathbb{D}}_{\infty}\right)$ through the transformation

$$
\mathcal{F} \circ\left(\beta^{-1}\right)^{*}=\left(\beta^{-1}\right)^{*} \circ \operatorname{Ad}_{\beta^{*}}(\mathcal{F}): \mathcal{O}\left(\widehat{\mathbb{D}}_{\infty}\right) \rightarrow \mathcal{O}\left(X_{0}\right),
$$

where $\operatorname{Ad}_{\beta^{*}}(\mathcal{F}):=\beta^{*} \circ \mathcal{F} \circ\left(\beta^{-1}\right)^{*}: \mathcal{O}\left(\widehat{\mathbb{D}}_{\infty}\right) \rightarrow \mathcal{O}(\widehat{\mathbb{C}} \backslash f(\mathbb{D}))$. A direct calculation shows that for each $h(\eta)=\sum_{k=0}^{\infty} a_{k} \eta^{-k} \in \mathcal{O}\left(\widehat{\mathbb{D}}_{\infty}\right)$, we have

$$
\left(\operatorname{Ad}_{\beta^{*}}(\mathcal{F})[h]\right)(w)=\frac{1}{2 \pi i} \int_{\partial f(\mathbb{D})} \frac{h\left(f^{-1}(\zeta)\right)}{1-\zeta w^{-1}} \frac{\mathrm{d} \zeta}{\zeta}, \quad w \in \widehat{\mathbb{C}} \backslash f(\mathbb{D}) .
$$

As a result, $\left(\operatorname{Ad}_{\beta^{*}}(\mathcal{F})[h]\right)(w)$ is a power series in $1 / w$. Actually, in view of the Cauchy integral formula

$$
\frac{1}{2 \pi i} \int_{S^{1}} \frac{\zeta^{n}}{1-\zeta \eta^{-1}} \frac{\mathrm{d} \zeta}{\zeta}=\left\{\begin{array}{ll}
\eta^{n} & \text { if } n \leqslant 0, \\
0 & \text { if } n \geqslant 1,
\end{array} \quad \eta \in \mathbb{D}_{\infty}\right.
$$

we have

$$
\left(\operatorname{Ad}_{\beta^{*}}(\mathcal{F})[h]\right)(w)=\sum_{k=0}^{n} \frac{a_{k}}{2 \pi i} \int_{\partial X_{0}} \frac{\left(f^{-1}(\zeta)\right)^{-k}}{1-\zeta w^{-1}} \frac{\mathrm{d} \zeta}{\zeta}=\sum_{k=0}^{n} a_{k}\left[\left(f^{-1}(w)\right)^{-k}\right]_{\leqslant 0},
$$


where $\left[\left(f^{-1}(w)\right)^{-k}\right]_{\leqslant 0}$ denotes the constant-part plus the principal-part of the Laurent series for $\left(f^{-1}(w)\right)^{-k}=\left(1 / f^{-1}(w)\right)^{k}$; hence every element in $\mathcal{O}(\widehat{\mathbb{C}} \backslash f(\mathbb{D}))$ can be written as a series in $1 / w$. The quantity

$$
Q_{k}(w):=\frac{1}{2 \pi i} \int_{\partial X_{0}} \frac{\left(f^{-1}(\zeta)\right)^{-k}}{1-\zeta w^{-1}} \frac{\mathrm{d} \zeta}{\zeta}=\left[\left(f^{-1}(w)\right)^{-k}\right]_{\leqslant 0},
$$

for $k \in \mathbb{N}$, is called the $k$-th Faber polynomial associated to the domain $\mathbb{C} \backslash \overline{f(\mathbb{D})}$ (or simply to $f$ ), and it is a polynomial of degree $k$ in $1 / w$, cf. also Section 2.4.

We conclude that $\left[\left(\beta^{-1}\right)^{*} \circ \operatorname{Ad}_{\beta^{*}}(h)\right] \circ(1 / z)^{-1}=\left[\operatorname{Ad}_{\beta^{*}}(h)\right] \circ f \circ \beta^{-1}$, and hence

$$
W_{f}=\overline{\operatorname{span}\left(\{1\} \cup\left\{\left.Q_{n} \circ f \circ(1 / z)\right|_{S^{1}}\right\}_{n \geqslant 1}\right)}{ }^{H},
$$

where $z$ is the identity map on $\widehat{\mathbb{D}}_{\infty}$; note, if $f(z) \equiv z$ then $W_{f}=H_{+}$.

\section{Remark 3.4.}

(a) The Faber polynomials appeared first (with a different formalism, but equivalent to our presentation) in the context of approximations of functions in one complex variable by analytic functions (see [8] and [9]). Since then, they also play an important role in the theory of univalent functions (see [30]). We introduced the Faber polynomials in a slightly non-standard way in order to have them in a form which is suitable for embedding univalent functions into the Grassmannian by using Faber polynomials.

(b) In the context of Abelian function theory, the exterior derivatives

$$
\omega_{\infty}^{(n)}:=\mathrm{d} Q_{n}(f(1 / z))
$$

$n=1,2, \ldots$ are known as Abelian differentials of the second kind on the Riemann sphere. In general, Krichever's embedding of the algebro-geometric datum $(X, \mathcal{O}, Q, z, \varphi)$, where

$$
\left(X, \alpha_{1}, \ldots, \alpha_{g}, \beta_{1}, \ldots, \beta_{g}\right)
$$

is a homologically marked compact Riemann surface with genus $g, \mathcal{O}$ is the structure sheaf of $X, Q \in X, z$ and $\varphi$ are local uniformisers, and a local trivialisation of $\mathcal{O}$, is described by using multivalued meromorphic functions $\varphi^{(0)}(z) \equiv 1$,

$$
\varphi^{(n)}(z):=\int^{z} \omega_{Q}^{(n)}=: z^{n}-\sum_{m=1}^{\infty} q_{n m} \frac{z^{-m}}{m},
$$

(modulo periods) where $\omega_{Q}^{(n)}$ 's are (normalised) abelian differentials of the second kind [14, Section 2.27 and p. 304]. These multivalued meromorphic functions can be regarded as a generalisation of the Faber polynomials (see [37, p. 131]).

(c) Given again a homologically marked compact Riemann surface $\left(X,\left(\alpha_{i}, \beta_{i}\right)_{i=1}^{g}\right)$ with genus $g$, Krichver's embedding of yet another datum $\left(X, \Omega^{1 / 2}, Q, z, \sqrt{\mathrm{d} z}\right)$ or

$$
\left(X, \Omega^{1 / 2} \otimes \mathcal{L}_{c}, Q, z, \sqrt{\mathrm{d} z} \otimes s_{c}\right)
$$

is described in [14, equation (2.34)]. Here, $\Omega^{1 / 2}$ is the so-called theta characteristic of the compact Riemann surface $X, \mathcal{L}_{c}$ is a complex line bundle of degree 0 parametrised by $c \in \mathbb{C}^{g}$ (modulo the lattice associated to $\left.\left(\alpha_{i}, \beta_{i}\right)_{i=1}^{g}\right)$, and $s_{c}$ is a local trivialisation of $\mathcal{L}_{c}$. In particular, the embedding of the latter and the associated Fermionic state (the image under the Plücker embedding) are described by means of the Szegö kernel of $\Omega^{1 / 2} \otimes \mathcal{L}_{c}$ (see $[1,14]$, in which, the scattering operator in [14, Section 5.12] is a special case of a Bogoliubov transformation discussed in [1, equations (2.15)-(2.20)]), and then the corresponding taufunction $\tau(\mathbf{t})$ is described as a theta function multiplied by $\exp \left(\sum_{n, m=1}^{\infty} q_{n m} t_{n} t_{m}\right)$ (see $[14$, Theorem 5.6]). 


\subsection{Action of words in Witt algebra generators}

Let $X=\left\{x_{1}, x_{2}, x_{3}, \ldots\right\}$ be an alphabet, consisting of a countable set of non-commuting letters. The free monoid $X^{*}$ on $X$ is the set of all words in the letters $X$, including the empty word $\varnothing$. We denote by

$$
\mathbb{C}\langle X\rangle:=\bigoplus_{w \in X^{*}} \mathbb{C} w=\mathbb{C} \oplus \bigoplus_{n=1}^{\infty} \mathbb{C}\langle X\rangle_{n}
$$

the free associative and unital $\mathbb{C}$-algebra on $X$. The unit of this algebra is the empty word which we will denote by $1:=\varnothing$. The set $\mathbb{C}\langle X\rangle_{n}$ stands for $\bigoplus_{|w|=n} \mathbb{C} w$ where the summation is taken over all words $w$ of length $n$.

Definition 3.5. We define

$$
\xi(\mathbf{x}, z):=\sum_{n=1}^{\infty} x_{k} z^{k} \in \mathbb{C}\langle X\rangle \llbracket z \rrbracket,
$$

and a distinguished element $S(\xi(\mathbf{x}, z)) \in \mathbb{C}\langle X\rangle \llbracket z \rrbracket$ by

$$
S(\xi(\mathbf{x}, z)):=1+\sum_{n=1}^{\infty} z^{n} \sum_{p=1}^{n} \sum_{\substack{i_{1}, \ldots, i_{p} \in \mathbb{N}: \\ i_{1}+\cdots+i_{p}=n}} x_{i_{1}} \cdots x_{i_{p}}
$$

Definition 3.6. Let $x_{0}:[0,+\infty) \rightarrow \mathbb{R}$ and $x_{1}, x_{2}, \ldots:[0,+\infty) \rightarrow \mathbb{C}$ be continuous and of bounded variation. For $0 \leqslant s \leqslant t$, we define $\left[\int 1\right]_{s, t}:=1$ and

$$
\left[\int\left(x_{i_{p}} \cdots x_{i_{2}} x_{i_{1}}\right)\right]_{s, t}:=\int_{s \leqslant u_{1}<u_{2}<\cdots<u_{p} \leqslant t} \mathrm{e}^{-i_{1} x_{0}\left(u_{1}\right)} \mathrm{d} x_{i_{1}}\left(u_{1}\right) \mathrm{e}^{-i_{2} x_{0}\left(u_{2}\right)} \mathrm{d} x_{i_{2}}\left(u_{2}\right) \cdots \mathrm{e}^{-i_{p} x_{0}\left(u_{p}\right)} \mathrm{d} x_{i_{p}}\left(u_{p}\right) .
$$

The action of $\int$ naturally extends to $\mathbb{C}\langle X\rangle \llbracket z \rrbracket$, and then we call

$$
S(\xi(\mathbf{x}, z))_{s, t}:=\left[\int S(\xi(\mathbf{x}, z))\right]_{s, t},
$$

the signature of $\xi(\mathbf{x}, z)$.

We define a bilinear map $T: \mathbb{C}\langle X\rangle\left(\left(z^{-1}\right)\right) \times \mathbb{C}\langle X\rangle \rightarrow \mathbb{C}\langle X\rangle\left(\left(z^{-1}\right)\right)$, by extending the pairing $T(f, 1):=f$, and $T\left(f, x_{i_{p}} \cdots x_{i_{1}}\right):=\left(L_{-i_{1}} \cdots L_{-i_{p}} f\right) x_{i_{p}} \cdots x_{i_{1}}$, bilinearly, for $f \in \mathbb{C}\langle X\rangle\left(\left(z^{-1}\right)\right)$, $p \geqslant 1$, and $i_{1}, \ldots, i_{p} \in \mathbb{N}$. Further, $L_{k}:=-z^{k+1} \partial /(\partial z)$, for $k \leqslant-1$, forms the negative part of the Witt algebra, cf. (1.1), and $\partial /(\partial z)$ is a formal derivation on $\mathbb{C}\langle X\rangle\left(\left(z^{-1}\right)\right)$.

For $f \in \mathbb{C}\langle X\rangle\left(\left(z^{-1}\right)\right)$ and $x \in \mathbb{C}\langle X\rangle$, in the sequel, $T(f, x)$, will be denoted by $f_{\cdot z} x$. The following is clear by definition:

Proposition 3.7. $T$ defines an action of the $\mathbb{C}$-algebra $\mathbb{C}\langle X\rangle$ on $\mathbb{C}\langle X\rangle\left(\left(z^{-1}\right)\right)$ from the right.

The right action $T$ can be extended to the right action

$$
\mathbb{C}\langle X\rangle\left(\left(w^{-1}\right)\right) \times \mathbb{C}\langle X\rangle \llbracket z \rrbracket \rightarrow \mathbb{C}\langle X\rangle\left(\left(w^{-1}\right)\right) \llbracket z \rrbracket,
$$

under which the image of $\left(f, z^{n} x_{i_{p}} \cdots x_{i_{1}}\right)$ is mapped to $z^{n}\left(f_{\cdot w} x_{i_{p}} \cdots x_{i_{1}}\right)=: f_{\cdot w}\left(z^{n} x_{i_{p}} \cdots x_{i_{1}}\right)$. Note that now the notation $f_{\cdot w} S(\mathbf{x})$ makes sense. 
Theorem 3.8. Let $\left\{f_{t}\right\}_{t \geqslant 0}$ be a solution to the Loewner-Kufarev equation. Then

$$
f_{t}(z)=\left[\int \operatorname{Res}_{w=0}\left(\frac{\mathrm{e}^{x_{0}(t)} z}{1-z w}\left(w^{-1} \cdot w S\left(\xi\left(\mathbf{x}, \mathrm{e}^{x_{0}(t)}\right)\right)\right)\right)\right]_{0, t} .
$$

Proof. By setting

$$
\widetilde{w}(n)_{i_{1}, \ldots, i_{p}}:=\left\{\left(n-i_{1}\right)+1\right\}\left\{\left(n-\left(i_{1}+i_{2}\right)\right)+1\right\} \cdots\left\{\left(n-\left(i_{1}+i_{2}+\cdots+i_{p-1}\right)\right)+1\right\},
$$

where $n=i_{1}+\cdots+i_{p}$, we have

$$
\begin{aligned}
& w^{-1} \cdot{ }_{w} 1=w^{-1}, \\
& w^{-1}{ }_{\cdot w} x_{i_{p}} \cdots x_{i_{1}}=\widetilde{w}(n)_{i_{1}, \ldots, i_{p}} x_{i_{p}} \cdots x_{i_{1}} w^{-\left(i_{1}+\cdots+i_{p}+1\right)} .
\end{aligned}
$$

Therefore $\operatorname{Res}_{w=0}\left(\sum_{m=0}^{\infty} z^{m} w^{m}\left(w^{-1} \cdot w 1\right)\right)=1$ (i.e., the empty word $\varnothing$ ), and

$$
\operatorname{Res}_{w=0}\left(\sum_{m=0}^{\infty} z^{m} w^{m}\left(w^{-1} \cdot w x_{i_{p}} \cdots x_{i_{1}}\right)\right)=z^{\left(i_{1}+\cdots+i_{p}\right)} \widetilde{w}(n)_{i_{1}, \ldots, i_{p}} x_{i_{p}} \cdots x_{i_{1}} .
$$

Hence we get

$$
\begin{aligned}
\operatorname{Res}_{w=0} & \left(\frac{\mathrm{e}^{x_{0}(t)} z}{1-z w}\left(w^{-1} \cdot w S\left(\xi\left(\mathbf{x}, \mathrm{e}^{x_{0}(t)}\right)\right)\right)\right) \\
= & \mathrm{e}^{x_{0}(t)} z+\sum_{n=1}^{\infty} \mathrm{e}^{(n+1) x_{0}(t)} z^{n+1} \sum_{p=1}^{n} \sum_{\substack{i_{1}, \ldots, i_{p} \in \mathbb{N}: \\
i_{1}+\cdots+i_{p}=n}} \widetilde{w}(n)_{i_{1}, \ldots, i_{p}} x_{i_{p}} \cdots x_{i_{1}} .
\end{aligned}
$$

Now, in view of Theorem 2.10, we obtain the result.

By tensoring the right action (3.2) this gives rise to

$$
\left(\mathbb{C}\langle X\rangle\left(\left(w^{-1}\right)\right) \otimes \mathbb{C}\langle X\rangle\left(\left(u^{-1}\right)\right)\right) \times(\mathbb{C}\langle X\rangle \llbracket z \rrbracket \otimes \mathbb{C}\langle X\rangle \llbracket z \rrbracket) \rightarrow \mathbb{C}\langle X\rangle\left(\left(w^{-1}\right)\right) \otimes \mathbb{C}\langle X\rangle\left(\left(u^{-1}\right)\right),
$$

under which the image of $(f \otimes g, x \otimes y)$ will be denoted by $\left(f_{\cdot w} x\right) \otimes(g \cdot u)$ in the sequel.

We recall (see [32, Proposition 3.3 and pp. 50-51]) that the tau-function corresponding to $W \in \mathrm{Gr}$, is given by

$$
\tau_{W}(\mathbf{t})=\operatorname{det}\left(w_{+}\right)=\operatorname{det}\left(1+a^{-1} b A\right),
$$

up to a multiplicative constant, where $w_{+}: \mathrm{e}^{\xi(\mathbf{t}, z)} W \rightarrow H_{+}$, is the orthogonal projection, and $\mathrm{e}^{\xi(\mathbf{t}, z)}: H \rightarrow H$, is the multiplication operator by $\mathrm{e}^{\xi(\mathbf{t}, z)}$, with matrix representation

$$
\mathrm{e}^{-\xi(\mathbf{t}, z)}=\left(\begin{array}{ll}
a & b \\
0 & d
\end{array}\right) \quad \text { along } H=H_{+} \oplus H_{-},
$$

and $A: H_{+} \rightarrow H_{-}$is such that $\operatorname{graph}(A)=W$. Given a bounded univalent function $f: \mathbb{D} \rightarrow \mathbb{C}$, with $f(0)=0$, we denote by $A_{f}: H_{+} \rightarrow H_{-}$the linear map such that $\operatorname{graph}\left(A_{f}\right)=W_{f}$.

Theorem 3.9. Let $\left\{f_{t}\right\}_{0 \leqslant t \leqslant T}$ be a univalent solution to the Loewner-Kufarev equation such that $f_{t}(\mathbb{D})$ is bounded for every $t \in[0, T]$. Then for each $h \in H_{+}$and $|z|>1$, we have

$$
\begin{aligned}
\left(A_{f_{t}} h\right)(z)= & {\left[\int \operatorname { R e s } _ { \substack { w = 0 , \\
u = 0 } } \left(\frac{h^{\prime}(u)}{w-z} \sum_{r, s=1}^{\infty} \mathrm{e}^{(r+s) x_{0}(t)} x_{r+s}\left(w^{-r} \cdot w S\left(\xi\left(\mathbf{x}, \mathrm{e}^{x_{0}(t)}\right)\right)\right)\right.\right.} \\
& \left.\left.\uplus\left(u^{-s} \cdot{ }_{u} S\left(\xi\left(\mathbf{x}, \mathrm{e}^{x_{0}(t)}\right)\right)\right)\right)\right]_{0, t} .
\end{aligned}
$$


The proof can be found in Appendix A.3. From this, we obtain

Corollary 3.10. For each $n, m \in \mathbb{N}$, the coefficient $b_{-n,-m}(t)$, is equal to

$$
\begin{gathered}
{\left[\int \operatorname { R e s } _ { \substack { z = 0 , \\
u = 0 } } \left\{\operatorname{Res}_{w=0} \frac{z^{m-1} u^{n-1}}{w-z} \sum_{r, s=1}^{\infty} \mathrm{e}^{(r+s) x_{0}(t)} x_{r+s}\left(w^{-r} \cdot{ }_{w} S\left(\xi\left(\mathbf{x}, \mathrm{e}^{x_{0}(t)}\right)\right)\right)\right.\right.} \\
\left.\left.\uplus\left(u^{-s} \cdot u S\left(\xi\left(\mathbf{x}, \mathrm{e}^{x_{0}(t)}\right)\right)\right)\right\}\right]_{0, t} .
\end{gathered}
$$

\section{A Appendix}

\section{A.1 Proof of Theorem 2.10}

By applying variation of constants to (2.5), we obtain the following recurence relation

$$
c_{n}(t)=\mathrm{e}^{n x_{0}(t)} \int_{0}^{t} \mathrm{e}^{-n x_{0}(s)} \mathrm{d} x_{n}(s)+\sum_{k=1}^{n-1}(k+1) \mathrm{e}^{n x_{0}(t)} \int_{0}^{t} \mathrm{e}^{-n x_{0}(s)} c_{k}(s) \mathrm{d} x_{n-k}(s),
$$

for $n \geqslant 2$. Multiplying by $\mathrm{e}^{-n x_{0}(t)}$, this transforms to

$$
\mathrm{e}^{-n x_{0}(t)} c_{n}(t)=\int_{0}^{t} \mathrm{e}^{-n x_{0}(t)} \mathrm{d} x_{n}(s)+\sum_{k=1}^{n-1}(k+1) \int_{0}^{t} \mathrm{e}^{-(n-k) x_{0}(t)} \mathrm{d} x_{n-k}(s)\left(\mathrm{e}^{-k x_{0}(s)} c_{k}(s)\right) .
$$

By assuming that $x_{1}, x_{2}, \ldots$ are non-commutative indeterminates, and the $c_{n}$ 's are polynomials in the $x_{i}$ 's, we shall consider the following equation:

$$
c_{n}=x_{n}+2 x_{n-1} c_{1}+3 x_{n-2} c_{2}+\cdots+(n-1) x_{2} c_{n-2}+n x_{1} c_{n-1},
$$

for $n \geqslant 1$ (roughly speaking, the polynomial $c_{n}$ means $\mathrm{e}^{-n x_{0}(t)} c_{n}(t)$ and 'applying the indeterminate $x_{k}$ from the left' means 'applying $\int_{0}^{t} \mathrm{e}^{-k x_{0}(s)} \mathrm{d} x_{k}(s) \times$ to functions of $s^{\prime}$ ) and then we shall make some observations about the equation (A.1) and introduce some notations: If we apply (A.1) to $c_{n}$, we get

(a) The terms $(n-k+1) x_{k} c_{n-k}$ for each $k=1,2, \ldots, n$. We shall denote these situation by

$$
c_{n} \stackrel{\widetilde{w}_{n, k} x_{k}}{\rightarrow} c_{n-k}
$$

respectively (note that the multiplication by the $x_{*}$ 's must sit just left to the next $c_{*}$ 's), where $\widetilde{w}_{n, k}:=((n-k)+1)$.

(b) The term $x_{0}$, to which we can not apply (A.1) anymore. This means, consider the situation that we apply (A.1) iteratively to $c_{*}$ 's which appeared at a previous stage. Suppose we have the term $c_{n}$ at some stage. Then chasing the term multiplied by $x_{*}$ which arose from the first term on the right-hand side in (A.1), lets us to get out of the loop of iterations; we shall symbolise this situation by

$$
c_{n} \stackrel{x_{n}}{\rightrightarrows} \text { end. }
$$

Let $p \in \mathbb{N}$ be such that $1 \leqslant p \leqslant n$. We fix $i_{1}, \ldots, i_{p} \in \mathbb{N}$, so that $i_{1}+\cdots+i_{p}=n$. This data permits one to get out of the loop of iterations of (A.1) as the following diagram shows:

$$
\begin{gathered}
c_{n} \stackrel{\widetilde{w}_{n, i_{1}} x_{i_{1}}}{\longrightarrow} c_{n-i_{1}} \stackrel{\widetilde{w}_{n-i_{1}, i_{2}} x_{i_{2}}}{\longrightarrow} c_{n-i_{1}-i_{2}} \stackrel{\widetilde{w}_{n-i_{1}-i_{2}, i_{3}} x_{i_{3}}}{\longrightarrow} \cdots \stackrel{\widetilde{w}_{n-\left(i_{1}+\cdots+i_{p-2}\right), i_{p-1}} x_{i_{p-1}}}{\longrightarrow} c_{n-\left(i_{1}+i_{2}+\cdots+i_{p-1}\right)} \\
=c_{i_{p}} \stackrel{x_{i_{p}}}{\longrightarrow} \text { end. }
\end{gathered}
$$


Hence we have a single path from $c_{n}$ to the 'end' in the above diagram. This path produces at the 'end' the term

$$
\widetilde{w}(n)_{i_{1}, \ldots, i_{p}} x_{i_{p}} x_{i_{p-1}} \cdots x_{i_{2}} x_{i_{1}}
$$

where, by using the relation $\widetilde{w}_{n-k, l}=\widetilde{w}_{n, k+l}$, the coefficient $\widetilde{w}(n)_{i_{1}, \ldots, i_{p}}$ is given by

$$
\begin{aligned}
\widetilde{w}(n)_{i_{1}, \ldots, i_{p}} & =\widetilde{w}_{n, i_{1}} \widetilde{w}_{n-i_{1}, i_{2}} \widetilde{w}_{n-i_{1}-i_{2}, i_{3}} \cdots \widetilde{w}_{n-\left(i_{1}+i_{2}+\cdots+i_{p-2}\right), i_{p-1}} \\
& =\widetilde{w}_{n, i_{1}} \widetilde{w}_{n, i_{1}+i_{2}} \widetilde{w}_{n, i_{1}+i_{2}+i_{3}} \cdots \widetilde{w}_{n, i_{1}+i_{2}+\cdots+i_{p-2}+i_{p-1}} \\
& =\left\{\left(n-i_{1}\right)+1\right\}\left\{\left(n-\left(i_{1}+i_{2}\right)\right)+1\right\} \cdots\left\{\left(n-\left(i_{1}+i_{2}+\cdots+i_{p-1}\right)\right)+1\right\} .
\end{aligned}
$$

Collecting all possibilities, we have

$$
c_{n}=\sum_{p=1}^{n} \sum_{\substack{i_{1}, \ldots, i_{p} \in \mathbb{N}: \\ i_{1}+\cdots+i_{p}=n}} \widetilde{w}(n)_{i_{1}, \ldots, i_{p}} x_{i_{p}} x_{i_{p-1}} \cdots x_{i_{2}} x_{i_{1}},
$$

which yields the result by reinterpreting it in the language of paths $x_{k}(t)$ 's, as claimed.

\section{A.2 Proof of Proposition 2.14}

By applying variation of constants to (2.8), we have

$$
\begin{aligned}
b_{-m,-n}(t)= & -\mathrm{e}^{(n+m) x_{0}(t)} \int_{0}^{t} \mathrm{e}^{-(n+m) x_{0}(t)} \mathrm{d} x_{n+m}(s) \\
& +\mathrm{e}^{(n+m) x_{0}(t)} \int_{0}^{t}\left\{(n-1) b_{-m,-(n-1)}(s) \mathrm{d} x_{1}(s)+\cdots+b_{-m,-1}(s) \mathrm{d} x_{n-1}(s)\right\} \\
& +\mathrm{e}^{(n+m) x_{0}(t)} \int_{0}^{t}\left\{(m-1) b_{-(m-1),-n}(s) \mathrm{d} x_{1}(s)+\cdots+b_{-1,-n}(s) \mathrm{d} x_{m-1}(s)\right\} .
\end{aligned}
$$

By assuming that $x_{1}, x_{2}, \ldots$ are non-commutative indeterminates, and the $b_{-m,-n}$ 's polynomials in the $x_{i}$ 's, we shall consider the following equation:

$$
\begin{aligned}
b_{-m,-n}= & -x_{n+m}+\left\{(n-1) b_{-m,-(n-1)} x_{1}+\cdots+2 b_{-m,-2} x_{n-2}+b_{-m,-1} x_{n-1}\right\} \\
& +\left\{(m-1) b_{-(m-1),-n} x_{1}+\cdots+2 b_{-2,-n} x_{m-2}+b_{-1,-n} x_{m-1}\right\},
\end{aligned}
$$

(roughly speaking, the polynomial $b_{-m,-n}$ means $\mathrm{e}^{-(m+n) x_{0}(t)} b_{-m,-n}(t)$ and 'applying the indeterminate $x_{k}$ from the right' means 'applying $\int_{0}^{t} \mathrm{e}^{-k x_{0}(s)} \mathrm{d} x_{k}(s) \times$ to functions of $\left.s^{\prime}\right)$. If we apply (A.2) to $b_{-m,-n}$, we get:

(a) The terms $(n-k) b_{-m,-(n-k)} x_{k}$ and $(m-k) b_{-(m-k),-n} x_{k}$ for each $k$. We shall denote these cases by

$$
b_{-m,-n} \stackrel{(n-k) x_{k} \times}{\rightarrow} b_{-m,-(n-k)} \quad \text { and } \begin{gathered}
b_{-m,-n} \\
(m-k) x_{k} \times \downarrow \\
b_{-(m-k),-n}
\end{gathered},
$$

respectively (Note that the multiplication by the $x_{*}$ 's must sit just right to the next $b_{*, *}$ 's).

(b) The term $-x_{n+m}$, to which we can not apply (A.2) anymore. This means, consider the situation that we apply (A.2) iteratively to the $b_{*, *}$ 's which appeared at a previous stage. Suppose that we have the term $b_{-m,-n}$ at some stage. Then chasing the term, multiplied 
by $-x_{*}$, which arose from the first term on the right-hand side in (A.2), permits us to get out of the loop of iterations. We shall denote this situation by

$$
b_{-m,-n} \stackrel{-x_{n+m} \times}{\rightrightarrows} \text { end } \quad \text { or } \quad \begin{gathered}
b_{-m,-n} \\
-x_{n+m} \times \underset{\text { end }}{\downarrow}
\end{gathered} .
$$

Note that the multiplication by the $x_{*}$ 's must be from the left. Hence in particular, to get the term of the form $x_{k}(\cdots)$ in the polynomial expression of $b_{-m,-n}$ in the $x_{i}$ 's, we have to escape the loop by passing to the cases

$$
b_{-i,-j} \stackrel{-x_{k} \times}{\rightrightarrows} \text { end } \quad \text { or } \quad \begin{gathered}
b_{-i,-j} \times \downarrow \downarrow \\
\text { end }
\end{gathered},
$$

where $i, j \in \mathbb{N}$ with $i+j=k$.

(c) If we have $b_{-1,-1}$, applying (A.2) does not produce $b_{*, *}$ 's. Namely we must have

$$
b_{-1,-1} \stackrel{-x_{2} \times}{\rightrightarrows} \text { end } \quad \text { or } \quad \begin{gathered}
-b_{-1,-1} \times \downarrow \downarrow \\
\text { end }
\end{gathered} .
$$

Again, the multiplication by $x_{2}$ must be from the left. In particular, $b_{-m,-n}$ does not contain the term $x_{1}(\cdots)$ and hence $b_{-m,-n}$ is a linear combination of $x_{k}(\cdots)$ 's for $k \geqslant 2$, though the factor $(\cdots)$ may involve $x_{1}$.

Let $k \in \mathbb{N}$ be such that $2 \leqslant k \leqslant n+m$. We shall find the term of the form $x_{k}(\cdots)$ in the polynomial expression of $b_{-m,-n}$ in the $x_{i}$ 's. For this, we shall fix $i \in\{1, \ldots, m\}$ and $j \in\{1, \ldots, n\}$ such that $i+j=k$. Suppose that $p, q \in \mathbb{N}$ and $i_{1}, \ldots, i_{p}, j_{1}, \ldots, j_{q} \in \mathbb{N}$ satisfy $i_{1}+\cdots+i_{p}=m-i$ and $j_{1}+\cdots+j_{q}=n-j$. We then put $a_{r}:=m-\left(i_{1}+\cdots+i_{r}\right)$ for $r=1, \ldots, p$ and $c_{s}:=n-\left(j_{1}+\cdots+j_{s}\right)$ for $s=1, \ldots, q$. Note that $a_{p}=i$ and $c_{q}=j$. According to this notation, we distinguish the following three cases:

(1) If there exist such $p, q,\left(i_{1}, \ldots, i_{p}\right)$ and $\left(j_{1}, \ldots, j_{q}\right)$, then we can consider the following diagram:

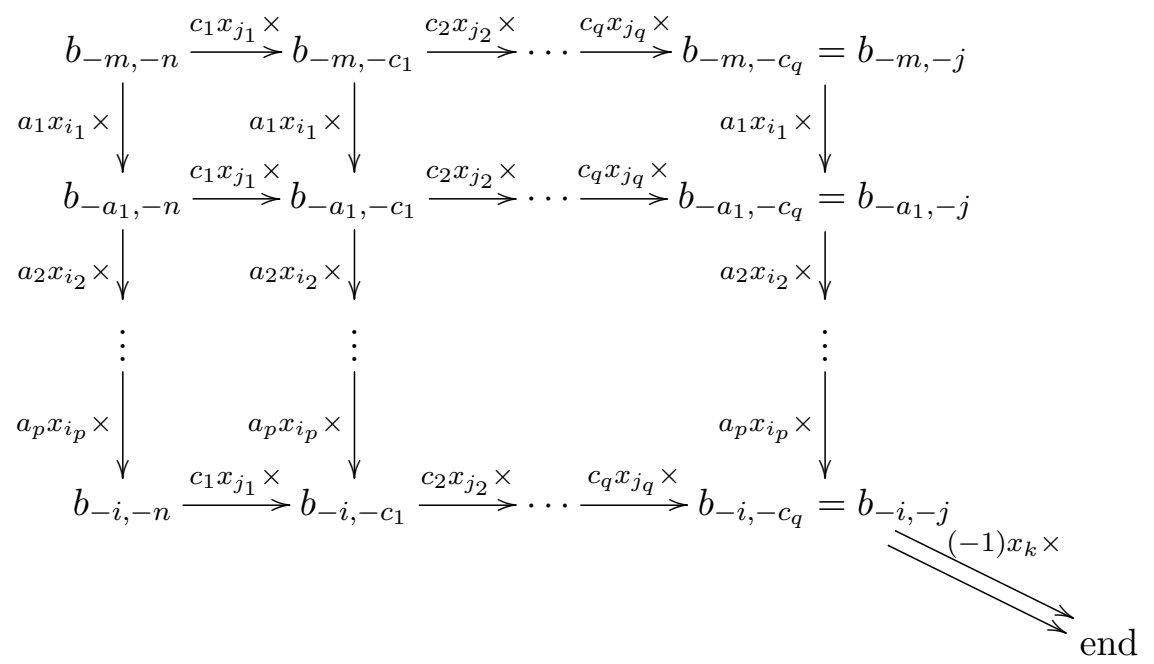

During the loop of iterations of (A.2), we have $\left(\begin{array}{c}p+q \\ p\end{array}\right)=\left(\begin{array}{c}p+q \\ q\end{array}\right)$-paths from $b_{-m,-n}$ to the 'end' in the above diagram, each of which produces terms

$$
-w_{i_{1}, \ldots, i_{p} ; j_{1}, \ldots, j_{q}} x_{k}(\cdots) \text { 's, }
$$


where

$$
\begin{aligned}
w_{i_{1}, \ldots, i_{p} ; j_{1}, \ldots, j_{q}}= & a_{1} a_{2} \cdots a_{p} b_{1} b_{2} \cdots b_{q} \\
= & \left(m-i_{1}\right)\left(m-\left(i_{1}+i_{2}\right)\right) \cdots\left(m-\left(i_{1}+i_{2}+\cdots+i_{p}\right)\right) \\
& \times\left(n-j_{1}\right)\left(n-\left(j_{1}+j_{2}\right)\right) \cdots\left(n-\left(j_{1}+j_{2}+\cdots+j_{q}\right)\right),
\end{aligned}
$$

(note that $w_{i_{1}, \ldots, i_{p} ; j_{1}, \ldots, j_{q}}$ depends only on $i_{1}, \ldots, i_{p}$ and $j_{1}, \ldots, j_{q}$ but not on the choice of paths in the diagram) and $(\cdots)$ is a monomial consisting of $x_{i_{p}}, x_{i_{p-1}}, \ldots, x_{i_{1}}$ and $x_{j_{q}}, x_{j_{q-1}}, \ldots, x_{j_{1}}$, which is interlacing according to a riffle shuffle permutation (note that we should distinguish, for example $x_{i_{1}} x_{j_{1}}$ and $x_{j_{1}} x_{i_{1}}$ even if $i_{1}=j_{1}$ ). Hence, in total all paths produce

$$
-w_{i_{1}, \ldots, i_{p} ; j_{1}, \ldots, j_{q}} x_{k}\left(\left(x_{i_{p}} x_{i_{p-1}} \cdots x_{i_{1}}\right) ш\left(x_{j_{q}} x_{j_{q-1}} \cdots x_{j_{1}}\right)\right) .
$$

(2) If there exist such a $p$ and $\left(i_{1}, \ldots, i_{p}\right)$ but not for $q$ and $\left(j_{1}, \ldots, j_{q}\right)$ (then we have $j=n$ ), then the diagram which we can have is the following:

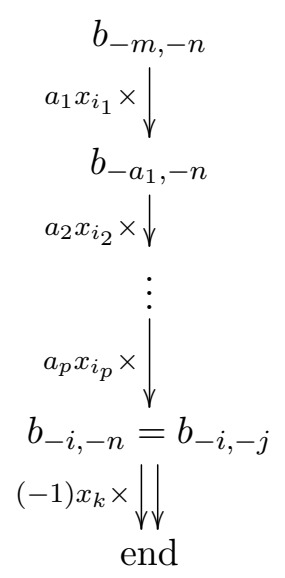

Hence we have a single path from $b_{-m,-n}$ to the 'end' in the above diagram. This path produces the term

$$
-w_{i_{1}, \ldots, i_{p}} x_{k}\left(x_{i_{p}} \cdots x_{i_{2}} x_{i_{1}}\right)
$$

where $w_{i_{1}, \ldots, i_{p} ; j_{1}, \ldots, j_{q}}=a_{1} a_{2} \cdots a_{p}=\left(m-i_{1}\right)\left(m-\left(i_{1}+i_{2}\right)\right) \cdots\left(m-\left(i_{1}+i_{2}+\cdots+i_{p}\right)\right)$.

(3) If there exist such a $q$ and $\left(j_{1}, \ldots, j_{q}\right)$ but not for $p$ and $\left(i_{1}, \ldots, i_{p}\right)$ (then we have $i=m$ ), then the diagram which we can have is the following:

$$
b_{-m,-n} \stackrel{c_{1} x_{j_{1}} \times}{\longrightarrow} b_{-m,-c_{1}} \stackrel{c_{2} x_{j_{2}} \times}{\longrightarrow} \cdots \stackrel{c_{q} x_{j_{q}} \times}{\longrightarrow} b_{-m,-c_{q}}=b_{-i,-j} \stackrel{(-1) x_{k} \times}{\longrightarrow} \text { end. }
$$

Hence we have a single path from $b_{-m,-n}$ to the 'end' in the above diagram. This path produces the term

$$
-w_{j_{1}, \ldots, j_{q}} x_{k}\left(x_{j_{q}} \cdots x_{j_{2}} x_{j_{1}}\right)
$$

where

$$
w_{j_{1}, \ldots, j_{q}}=c_{1} c_{2} \cdots c_{q}=\left(n-j_{1}\right)\left(n-\left(j_{1}+j_{2}\right)\right) \cdots\left(n-\left(j_{1}+j_{2}+\cdots+j_{q}\right)\right)
$$

Now by reinterpreting it in the language of paths $x_{k}(t)$ 's, we obtain the result. 


\section{A.3 Proof of Theorem 3.9}

Since $\left\{u^{n}\right\}_{n \geqslant 1}$ forms a basis of $H_{+}$, it is enough to show that

$$
\begin{aligned}
& n\left[\int \operatorname{Res}_{\substack{w=0, u=0}}\left(\frac{u^{n-1}}{w-z} \sum_{r, s=1}^{\infty} \mathrm{e}^{(r+s) x_{0}(t)} x_{r+s}\left(w^{-r} \cdot w S\left(\xi\left(\mathbf{x}, \mathrm{e}^{x_{0}(t)}\right)\right)\right) ш\left(u^{-s} \cdot u S\left(\xi\left(\mathbf{x}, \mathrm{e}^{x_{0}(t)}\right)\right)\right)\right)\right]_{t} \\
& \quad=n \sum_{m=1}^{\infty} b_{-n,-m}(t) z^{-m}
\end{aligned}
$$

where $b_{-n,-m}(t)$ are the Grunsky coefficients associated with $f_{t}$.

According to the decomposition

$$
S\left(\xi\left(\mathbf{x}, \mathrm{e}^{x_{0}(t)}\right)\right)=1+\sum_{m^{\prime}=1}^{\infty} \mathrm{e}^{m^{\prime} x_{0}(t)} \sum_{p=1}^{m^{\prime}} \sum_{\substack{i_{1}, \ldots, i_{p} \in \mathbb{N}: \\ i_{1}+\cdots+i_{p}=m^{\prime}}} x_{i_{1}} x_{i_{2}} \cdots x_{i_{p}},
$$

we have

$$
\begin{aligned}
& \left(w^{-r} \cdot w S\left(\xi\left(\mathbf{x}, \mathrm{e}^{x_{0}(t)}\right)\right)\right) \amalg\left(u^{-s} \cdot u S\left(\xi\left(\mathbf{x}, \mathrm{e}^{x_{0}(t)}\right)\right)\right) \\
& \quad=\left(w^{-r} \cdot{ }_{w} 1\right) \amalg\left(u^{-s} \cdot u 1\right)+F_{r, s}(w, u)+G_{r, s}(w, u)+H_{r, s}(w, u),
\end{aligned}
$$

where

$$
\begin{aligned}
& F_{r, s}(w, u):=\left[w^{-r} \cdot{ }_{w}\left(S\left(\xi\left(\mathbf{x}, \mathrm{e}^{x_{0}(t)}\right)\right)-1\right)\right] ш\left[u^{-s} \cdot u\left(S\left(\xi\left(\mathbf{x}, \mathrm{e}^{x_{0}(t)}\right)\right)-1\right)\right] \\
& =\sum_{m^{\prime}=1}^{\infty} \sum_{n^{\prime}=1}^{\infty} \sum_{p=1}^{m^{\prime}} \sum_{q=1}^{n^{\prime}} \sum_{\substack{i_{1}, \ldots, i_{p} \in \mathbb{N}: \\
i_{1}+\cdots+i_{p}=m^{\prime}}} \sum_{\substack{j_{1}, \ldots, j_{q} \in \mathbb{N}: \\
j_{1}+\cdots+j_{q}=n^{\prime}}} \mathrm{e}^{\left(m^{\prime}+n^{\prime}\right) x_{0}(t)} \\
& \times\left(w^{-r} \cdot{ }_{w} x_{i_{1}} x_{i_{2}} \cdots x_{i_{p}}\right) \amalg\left(u^{-s} \cdot{ }_{u} x_{j_{1}} x_{j_{2}} \cdots x_{j_{q}}\right), \\
& G_{r, s}(w, u):=\left(w^{-r} \cdot{ }_{w} 1\right) \amalg\left[u^{-s} \cdot u\left(S\left(\xi\left(\mathbf{x}, \mathrm{e}^{x_{0}(t)}\right)\right)-1\right)\right] \\
& =\sum_{n^{\prime}=1}^{\infty} \mathrm{e}^{n^{\prime} x_{0}(t)} \sum_{q=1}^{n^{\prime}} \sum_{\substack{j_{1}, \ldots, j_{q} \in \mathbb{N}: \\
j_{1}+\cdots+j_{q}=n^{\prime}}}\left(w^{-r} \cdot{ }_{w} 1\right) ш\left(u^{-s} \cdot u x_{j_{1}} x_{j_{2}} \cdots x_{j_{q}}\right), \\
& H_{r, s}(w, u):=\left[w^{-r} \cdot{ }_{w}\left(S\left(\xi\left(\mathbf{x}, \mathrm{e}^{x_{0}(t)}\right)\right)-1\right)\right] ш\left(u^{-s} \cdot u 1\right) \\
& =\sum_{m^{\prime}=1}^{\infty} \mathrm{e}^{m^{\prime} x_{0}(t)} \sum_{p=1}^{m^{\prime}} \sum_{\substack{i_{1}, \ldots, i_{p} \in \mathbb{N}: \\
i_{1}+\cdots+i_{p}=m^{\prime}}}\left(w^{-r} \cdot w x_{i_{1}} x_{i_{2}} \cdots x_{i_{p}}\right) \amalg\left(u^{-s} \cdot u 1\right) .
\end{aligned}
$$

Since $w^{-r} \cdot{ }_{w} 1=w^{-r}$, we get $\left(w^{-r} \cdot{ }_{w} 1\right) ш\left(u^{-s} \cdot u 1\right)=w^{-r} u^{-s}$. Then, by using

$$
\frac{1}{w-z}=-\sum_{m=1}^{\infty} z^{-m} w^{m-1} \quad \text { for }|z|>|w|,
$$

we have

$$
\operatorname{Res}_{\substack{w=0 ; \\ u=0}}\left(\frac{u^{n-1}}{w-z} \sum_{r, s=1}^{\infty} \mathrm{e}^{(r+s) x_{0}(t)} x_{r+s}\left(\left(w^{-r} \cdot{ }_{w} 1\right) ш\left(u^{-s} \cdot u 1\right)\right)\right)
$$




$$
\begin{aligned}
& =-\operatorname{Res}_{\substack{w=0 ; \\
u=0}}\left(u^{n-1} \sum_{m=1}^{\infty} z^{-m} w^{m-1} \sum_{r, s=1}^{\infty} \mathrm{e}^{(r+s) x_{0}(t)} x_{r+s} w^{-r} u^{-s}\right) \\
& =-\sum_{m=1}^{\infty} z^{-m} \mathrm{e}^{(m+n) x_{0}(t)} x_{m+n} .
\end{aligned}
$$

Let

$$
\begin{gathered}
w(r)_{i_{1}, \ldots, i_{p} ; \varnothing}:=r\left(i_{p}+r\right)\left(i_{p}+i_{p-1}+r\right) \cdots\left(i_{p}+i_{p-1}+\cdots+i_{2}+r\right) \\
=\left(m-\left(i_{1}+\cdots+i_{p}\right)\right) \cdots\left(m-\left(i_{1}+i_{2}\right)\right)\left(m-i_{1}\right),
\end{gathered}
$$

where $m=i_{1}+\cdots+i_{p}+r$,

$$
\begin{gathered}
w(s)_{\varnothing ; j_{1}, \ldots, j_{q}}:=s\left(j_{q}+s\right)\left(j_{q}+j_{q-1}+s\right) \cdots\left(j_{q}+j_{q-1}+\cdots+j_{2}+s\right) \\
=\left(n-\left(j_{1}+\cdots+j_{q}\right)\right) \cdots\left(n-\left(j_{1}+j_{2}\right)\right)\left(n-j_{1}\right),
\end{gathered}
$$

where $n=j_{1}+\cdots+j_{q}+s$, and

$$
w(r, s)_{i_{1}, \ldots, i_{p} ; j_{1}, \ldots, j_{q}}:=w(r)_{i_{1}, \ldots, i_{p} ; \varnothing} w(s)_{\varnothing ; j_{1}, \ldots, j_{q}} .
$$

For $F_{r+s}(w, u)$, we first observe that

$$
\begin{aligned}
w^{-r} \cdot{ }_{w} x_{i_{p}} \cdots x_{i_{2}} x_{i_{1}} & =x_{i_{p}} \cdots x_{i_{2}} x_{i_{1}} L_{-i_{1}} L_{-i_{2}} \cdots L_{-i_{p}} w^{-r} \\
& =w(r)_{i_{1}, \ldots, i_{p} ; \varnothing} x_{i_{p}} \cdots x_{i_{2}} x_{i_{1}} w^{-\left(i_{1}+i_{2}+\cdots+i_{p}+r\right)},
\end{aligned}
$$

and similarly

$$
u^{-s}{ }_{\cdot u} x_{j_{q}} \cdots x_{j_{2}} x_{j_{1}}=w(s)_{\varnothing ; j_{1}, \ldots, j_{q}} x_{j_{q}} \cdots x_{j_{2}} x_{j_{1}} u^{-\left(j_{1}+j_{2}+\cdots+j_{p}+s\right)} .
$$

Therefore we have

$$
\begin{aligned}
\operatorname{Res}_{\substack{w=0 ; \\
u=0}}( & \left.\frac{u^{n-1}}{w-z} x_{r+s}\left(\left(w^{-r} \cdot w x_{i_{1}} x_{i_{2}} \cdots x_{i_{p}}\right) \amalg\left(u^{-s} \cdot u x_{i_{1}} x_{i_{2}} \cdots x_{i_{p}}\right)\right)\right) \\
= & -1_{\left\{1 \leqslant n-s=j_{1}+\cdots+j_{q}\right\}} \sum_{m=1}^{\infty} z^{-m} 1_{\left\{1 \leqslant m-r=i_{1}+\cdots+i_{p}\right\}} \\
& \times w(r, s)_{i_{1}, \ldots, i_{p} ; j_{1}, \ldots, j_{q}} x_{r+s}\left[\left(x_{i_{p}} \cdots x_{i_{2}} x_{i_{1}}\right) \amalg\left(x_{j_{q}} \cdots x_{j_{2}} x_{j_{1}}\right)\right],
\end{aligned}
$$

so that

$$
\begin{aligned}
& \operatorname{Res}_{\substack{w=0 ; \\
u=0}}\left(\frac{u^{n-1}}{w-z} x_{r+s} F_{r, s}(w, u)\right) \\
& =-\sum_{m=1}^{\infty} z^{-m} \sum_{m^{\prime}=1}^{\infty} \sum_{n^{\prime}=1}^{\infty} \mathrm{e}^{\left(m^{\prime}+n^{\prime}\right) x_{0}(t)} \sum_{p=1}^{m^{\prime}} \sum_{q=1}^{n^{\prime}} \sum_{\substack{i_{1}, \ldots, i_{p} \in \mathbb{N}: \\
i_{1}+\cdots+i_{p}=m^{\prime}}} 1_{\left\{1 \leqslant m-r=i_{1}+\cdots+i_{p}\right\}} \cdots \\
& \cdots \sum_{\substack{j_{1}, \ldots, j_{q} \in \mathbb{N}: \\
j_{1}+\cdots+j_{q}=n^{\prime}}} 1_{\left\{1 \leqslant n-s=j_{1}+\cdots+j_{q}\right\}} w(r, s)_{i_{1}, \ldots, i_{p} ; j_{1}, \ldots, j_{q}} x_{r+s}\left[\left(x_{i_{p}} \cdots x_{i_{2}} x_{i_{1}}\right) \amalg\left(x_{j_{q}} \cdots x_{j_{2}} x_{j_{1}}\right)\right] \\
& =-1_{\{1 \leqslant n-s\}} \sum_{m=1}^{\infty} z^{-m} \mathrm{e}^{((m-r)+(n-s)) x_{0}(t)} 1_{\{1 \leqslant m-r\}} \sum_{p=1}^{m-r} \sum_{q=1}^{n-s} \sum_{\substack{i_{1}, \ldots, i_{p} \in \mathbb{N}: \\
i_{1}+\cdots+i_{p}=m-r}} \cdots
\end{aligned}
$$




$$
\cdots \sum_{\substack{j_{1}, \ldots, j_{q} \in \mathbb{N}: \\ j_{1}+\cdots+j_{q}=n-s}} w(r, s)_{i_{1}, \ldots, i_{p} ; j_{1}, \ldots, j_{q}} x_{r+s}\left[\left(x_{i_{p}} \cdots x_{i_{2}} x_{i_{1}}\right) \amalg\left(x_{j_{q}} \cdots x_{j_{2}} x_{j_{1}}\right)\right] .
$$

Hence we have reached

$$
\begin{aligned}
& \operatorname{Res}_{\substack{w=0 ; \\
u=0}}\left(\frac{u^{n-1}}{w-z} \sum_{r, s=1}^{\infty} \mathrm{e}^{(r+s) x_{0}(t)} x_{r+s} F_{r, s}(w, u)\right) \\
& =-\sum_{m=1}^{\infty} z^{-m} \mathrm{e}^{(m+n) x_{0}(t)} \sum_{k=2}^{m+n-2} \sum_{\substack{1 \leqslant r<m ; \\
1 \leqslant s<n: \\
r+s=k}} \sum_{p=1}^{m-r} \sum_{q=1}^{n-s} \sum_{\substack{i_{1}, \ldots, i_{p} \in \mathbb{N}: \\
i_{1}+\cdots+i_{p}=m-r}} \cdots \\
& \cdots \sum_{\substack{j_{1}, \ldots, j_{q} \in \mathbb{N}: \\
j_{1}+\cdots+j_{q}=n-s}} w(r, s)_{i_{1}, \ldots, i_{p} ; j_{1}, \ldots, j_{q}} x_{k}\left[\left(x_{i_{p}} \cdots x_{i_{2}} x_{i_{1}}\right) ш\left(x_{j_{q}} \cdots x_{j_{2}} x_{j_{1}}\right)\right] .
\end{aligned}
$$

Similarly, we find that

$$
\begin{aligned}
& \operatorname{Res}_{\substack{w=0 ; \\
u=0}}\left(\frac{u^{n-1}}{w-z} \sum_{r, s=1}^{\infty} \mathrm{e}^{(r+s) x_{0}(t)} x_{r+s} G_{r, s}(w, u)\right) \\
& =-\sum_{m=1}^{\infty} z^{-m} \sum_{r, s=1}^{\infty} \mathrm{e}^{(r+s) x_{0}(t)} \sum_{n^{\prime}=1}^{\infty} \mathrm{e}^{n^{\prime} x_{0}(t)} \sum_{q=1}^{n^{\prime}} \cdots \\
& \cdots \sum_{\substack{j_{1}, \ldots, j_{q} \in \mathbb{N}: \\
j_{1}+\cdots+j_{q}=n^{\prime}}} 1_{\left\{1 \leqslant n-s=j_{1}+\cdots+j_{q}\right\}} 1_{\{m=r\}} w(s)_{\varnothing ; j_{1}, \ldots, j_{q}} x_{r+s}\left(x_{j_{q}} \cdots x_{j_{1}}\right) \\
& =-\sum_{m=1}^{\infty} z^{-m} \mathrm{e}^{(m+n) x_{0}(t)} \sum_{k=m+1}^{m+n-1} \sum_{q=1}^{n+m-k} \sum_{\substack{j_{1}, \ldots, j_{q} \in \mathbb{N}: \\
j_{1}+\cdots+j_{q}=n+m-k}} w(k-m)_{\varnothing ; j_{1}, \ldots, j_{q}} x_{k}\left(x_{j_{q}} \cdots x_{j_{1}}\right) \text {, }
\end{aligned}
$$

and

$$
\begin{aligned}
\operatorname{Res}_{\substack{w=0 ; \\
u=0}}\left(\frac{u^{n-1}}{w-z} \sum_{r, s=1}^{\infty} \mathrm{e}^{(r+s) x_{0}(t)} x_{r+s} H_{r, s}(w, u)\right) \\
\quad=-\sum_{m=1}^{\infty} z^{-m} \mathrm{e}^{(m+n) x_{0}(t)} \sum_{k=n+1}^{m+n-1} \sum_{p=1}^{n+m-k} \sum_{\substack{i_{1}, \ldots, i_{p} \in \mathbb{N}: \\
i_{1}+\cdots+i_{p}=m+n-k}} w(k-n)_{i_{1}, \ldots, i_{p} ; \varnothing} x_{k}\left(x_{i_{p}} \cdots x_{i_{1}}\right) .
\end{aligned}
$$

Now, in view of Theorem 2.13, we obtain (A.3), and hence the result.

\section{Acknowledgements}

T.A. was supported by JSPS KAKENHI Grant Number 15K17562. R.F. was previously supported by the ERC advanced grant "Noncommutative distributions in free probability". Both authors thank Theo Sturm for the hospitality he offered to T.A. at the University of Bonn. T.A. thanks Roland Speicher for the hospitality offered him in Saarbrücken. R.F. thanks Roland Speicher for discussions, and Fukuoka University and the MPI in Bonn for their hospitality. We both thank Takuya Murayama for the discussions, comments and collaboration. We thank the anonymous referees for their comments which helped us to improve the paper. 


\section{References}

[1] Alvarez-Gaumé L., Gomez C., Moore G., Vafa C., Strings in the operator formalism, Nuclear Phys. B 303 (1988), 455-521.

[2] Amaba T., Friedrich R., Modulus of continuity of controlled Loewner-Kufarev equations and random matrices, Anal. Math. Phys. 10 (2020), 23, 29 pages, arXiv:1809.00536.

[3] Amaba T., Friedrich R., Murayama T., Univalence and holomorphic extension of the solution to $\omega$-controlled Loewner-Kufarev equations, J. Differential Equations 269 (2020), 2697-2704, arXiv:1909.13666.

[4] Baudoin F., An introduction to the geometry of stochastic flows, Imperial College Press, London, 2004.

[5] Bracci F., Contreras M.D., Díaz-Madrigal S., Vasil'ev A., Classical and stochastic Löwner-Kufarev equations, in Harmonic and Complex Analysis and its Applications, Trends Math., Birkhäuser/Springer, Cham, 2014, 39-134.

[6] Doyon B., Conformal loop ensembles and the stress-energy tensor, Lett. Math. Phys. 103 (2013), 233-284, arXiv:1209.1560.

[7] Duplantier B., Nguyen C., Nguyen N., Zinsmeister M., The coefficient problem and multifractality of wholeplane SLE \& LLE, Ann. Henri Poincaré 16 (2015), 1311-1395, arXiv:1211.2451.

[8] Ellacott S.W., A survey of Faber methods in numerical approximation, Comput. Math. Appl. Part B 12 (1986), 1103-1107.

[9] Faber G., Über polynomische Entwickelungen, Math. Ann. 57 (1903), 389-408.

[10] Friedrich R., The global geometry of stochastic Lœwner evolutions, in Probabilistic Approach to Geometry, Adv. Stud. Pure Math., Vol. 57, Math. Soc. Japan, Tokyo, 2010, 79-117, arXiv:0906.5328.

[11] Friedrich R., Kalkkinen J., On conformal field theory and stochastic Loewner evolution, Nuclear Phys. B 687 (2004), 279-302, arXiv:hep-th/0308020.

[12] Hidalgo R.A., Markina I., Vasil'ev A., Finite dimensional grading of the Virasoro algebra, Georgian Math. J. 14 (2007), 419-434.

[13] Johnston E., The Faber transform and analytic continuation, Proc. Amer. Math. Soc. 103 (1988), $237-243$.

[14] Kawamoto N., Namikawa Y., Tsuchiya A., Yamada Y., Geometric realization of conformal field theory on Riemann surfaces, Comm. Math. Phys. 116 (1988), 247-308.

[15] Kirillov A.A., Yuriev D.V., Representations of the Virasoro algebra by the orbit method, J. Geom. Phys. 5 (1988), 351-363.

[16] Kontsevich M., CFT, SLE and phase boundaries, Preprint, Arbeitstagung, MPI Bonn, 2003.

[17] Krichever I.M., Algebraic-geometric construction of the Zakharov-Shabat equations and their periodic solutions, Sov. Math. Dokl. 17 (1976), 394-397.

[18] Krichever I.M., Integration of nonlinear equations by the methods of algebraic geometry, Funct. Anal. Appl. 11 (1977), 12-26.

[19] Krichever I.M., Methods of algebraic geometry in the theory of non-linear equations, Russian Math. Surveys 32 (1977), no. 6, 185-213.

[20] Kufareff P.P., On one-parameter families of analytic functions, Math. Sb. 13(55) (1943), 87-118.

[21] Löwner K., Untersuchungen über schlichte konforme Abbildungen des Einheitskreises. I, Math. Ann. 89 (1923), 103-121.

[22] Lyons T.J., Caruana M., Lévy T., Differential equations driven by rough paths, Lecture Notes in Math., Vol. 1908, Springer, Berlin, 2007.

[23] Malliavin P., The canonic diffusion above the diffeomorphism group of the circle, C. R. Acad. Sci. Paris Sér. I Math. 329 (1999), 325-329.

[24] Markina I., Prokhorov D., Vasil'ev A., Sub-Riemannian geometry of the coefficients of univalent functions, J. Funct. Anal. 245 (2007), 475-492, arXiv:math.CV/0608532.

[25] Markina I., Vasil'ev A., Virasoro algebra and dynamics in the space of univalent functions, in Five Lectures in Complex Analysis, Contemp. Math., Vol. 525, Amer. Math. Soc., Providence, RI, 2010, 85-116.

[26] Markina I., Vasil'ev A., Löwner-Kufarev evolution in the Segal-Wilson Grassmannian, in Geometric Methods in Physics, Trends in Mathematics, Editors P. Kielanowski, S.T. Ali, A. Odzijewicz, M. Schlichenmaier, T. Voronov, Birkhäuser/Springer, Basel, 2013, 367-376. 
[27] Markina I., Vasil'ev A., Evolution of smooth shapes and integrable systems, Comput. Methods Funct. Theory 16 (2016), 203-229, arXiv:1108.1007.

[28] Mumford D., An algebro-geometric construction of commuting operators and of solutions to the Toda lattice equation, Korteweg deVries equation and related nonlinear equation, in Proceedings of the International Symposium on Algebraic Geometry (Kyoto Univ., Kyoto, 1977), Editor M. Nagata, Kinokuniya Book Store, Tokyo, 1978, 115-153.

[29] Pommerenke C., Univalent functions (with a chapter on quadratic differentials by Gerd Jensen), Studia Mathematica/Mathematische Lehrbücher, Vol. 25, Vandenhoeck \& Ruprecht, Göttingen, 1975.

[30] Schiffer M., Faber polynomials in the theory of univalent functions, Bull. Amer. Math. Soc. 54 (1948), 503-517.

[31] Schramm O., Scaling limits of loop-erased random walks and uniform spanning trees, Israel J. Math. 118 (2000), 221-288, arXiv:math.PR/9904022.

[32] Segal G., Wilson G., Loop groups and equations of KdV type, Inst. Hautes Études Sci. Publ. Math. 61 (1985), 5-65.

[33] Sola A., Elementary examples of Loewner chains generated by densities, Ann. Univ. Mariae CurieSktodowska Sect. A $\mathbf{6 7}$ (2013), 83-101.

[34] Takasaki K., Takebe T., SDiff(2) Toda equation - hierarchy, tau function, and symmetries, Lett. Math. Phys. 23 (1991), 205-214, arXiv:hep-th/9112042.

[35] Teo L.-P., Analytic functions and integrable hierarchies - characterization of tau functions, Lett. Math. Phys. 64 (2003), 75-92, arXiv:hep-th/0305005.

[36] Wiegmann P.B., Zabrodin A., Conformal maps and integrable hierarchies, Comm. Math. Phys. 213 (2000), 523-538, arXiv:hep-th/9909147.

[37] Yamada A., Precise variational formulas for abelian differentials, Kodai Math. J. 3 (1980), 114-143. 